\title{
Line Broadening in Field Metal-poor Red Giant and Red Horizontal Branch Stars
}

\author{
Bruce W. Carney \\ Department of Physics $\mathcal{E}$ Astronomy \\ University of North Carolina \\ Chapel Hill, NC 27599-3255 \\ e-mail:bruce@unc.edu \\ David W. Latham \& Robert P. Stefanik \\ Harvard-Smithsonian Center for Astrophysics \\ 60 Garden Street, Cambridge, MA 02138 \\ e-mail:dlatham@cfa.harvard.edu; rstefanik@cfa.harvard.edu \\ John B. Laird \\ Department of Physics $\mathcal{E}$ Astronomy \\ Bowling Green State University \\ Bowling Green, $\mathrm{OH} 43403$ \\ e-mail: laird@bgsu.edu
}

\begin{abstract}
We report 349 radial velocities for 45 metal-poor field red giant and red horizontal branch stars, with time coverage ranging from 1 to 21 years. We have identified one new spectroscopic binary, HD 4306, and one possible such system, HD 184711. We also report 57 radial velocities for 11 of the 91 stars reported on previously by Carney et al. (2003). All but one of the 11 stars had been found to have variable radial velocities. New velocities for the long-period spectroscopic binaries BD-1 2582 and HD 108317 have extended the time coverage to 21.7 and 12.5 years, respectively, but in neither case have we yet completed a full orbital period. As was found in the previous study, radial velocity "jitter" is present in many of the most luminous stars. Excluding stars showing spectroscopic binary orbital motion, all 7 of the red giants with estimated $M_{\mathrm{V}}$ values more luminous than -2.0 display jitter, as well as 3 of the 14 stars with $-2.0<M_{\mathrm{V}} \leq-1.4$. We have also measured the line broadening in all the new spectra, using synthetic spectra as templates. Comparison with results from high-resolution and higher signal-to-noise $(\mathrm{S} / \mathrm{N})$ spectra employed by other workers shows good agreement
\end{abstract}


down to line broadening levels of $3 \mathrm{~km} \mathrm{~s}^{-1}$, well below our instrumental resolution of $8.5 \mathrm{~km} \mathrm{~s}^{-1}$. As the previous work demonstrated, most of the most luminous red giants show significant line broadening, as do many of the red horizontal branch stars, and we discuss briefly possible causes. The line broadening appears related to velocity jitter, in that both appear primarily among the highest luminosity red giants.

Subject headings: binaries: spectroscopic — stars: kinematics — planetary systems — stars: Population II — stars: rotation — Galaxy: halo

\section{INTRODUCTION}

In a previous paper (Carney et al. 2003 hereafter C2003), we discussed results from over two thousand high-resolution $(\mathrm{R}=32,000)$, low signal-to-noise (10 to 50 per resolution element) spectra of 91 field metal-poor red giant branch (RGB) and red horizontal branch (RHB) stars. Radial velocities were obtained by cross-correlating each observed spectrum with a synthetic spectrum that closely matched the adopted temperature, gravity, and metallicity for the star. Sixteen stars were found to be single-lined spectroscopic binaries, and orbital solutions were presented for 14 of them. Excluding those 14 stars, observations of the stars covered spans from 2956 days to 6670 days, roughly from 8 to 18 years, with an average of 13.7 years.

The use of synthetic spectra enabled us to measure line broadening as well as radial velocities. We found some anticipated results as well as some surprises. For example, studies of RGB stars in globular clusters (Gunn \& Griffin 1979; Mayor \& Mermilliod 1984; Lupton et al. 1987; Pryor et al. 1988; Côté et al. 1996, 2002; Brown \& Wallerstein 1992; Kraft et al. 1997; Mayor et al. 1997) have shown that the most luminous stars, generally with $M_{\mathrm{V}} \leq-1.4$, show velocity variability that is unlikely to be associated with orbital motion. About half of the stars studied by $\mathrm{C} 2003$ with estimated $M_{\mathrm{V}}$ values more luminous than -1.4 also showed such velocity variability, which is often referred to as "jitter". The term's ambiguity describes the absence of a well-understood cause of the velocity variability.

As expected, the spectroscopic binary frequency, for periods less than 6000 days, was very similar to that found for metal-poor dwarfs and subgiants (Latham et al. 2002; Goldberg et al. 2002). There was some evidence for a dearth of short-period binaries among the RGB and RHB stars, which is not too surprising. Most short period systems are expected to undergo mass transfer when the more massive star begins to expand and fills its Roche lobe. The period does shorten initially, until the donor star's mass becomes less than that 
of the original secondary star, following which the orbital separation widens and the period increases. Among the fourteen spectroscopic binaries in C2003 with orbital solutions, two systems with small ratios of orbital semi-major axis to estimated primary stellar radius have circular orbits, presumably the result of tidal interactions. These two stars also had higher line broadenings than other RGB stars, presumably due to higher rotational velocities, a consequence of tidally-induced locking of the rotational and orbital periods 1 .

The most unexpected result involved the dependence of the measured line broadening on the stars' evolutionary state. For most of the RGB stars, the line broadening was generally smaller than our instrumental resolution (about $8.5 \mathrm{~km} \mathrm{~s}^{-1}$ ). But at the highest luminosities, the mean line broadening rose to values as high as about $12 \mathrm{~km} \mathrm{~s}^{-1}$ at $M_{\mathrm{V}} \approx-2.6$, and perhaps as high as $15 \mathrm{~km} \mathrm{~s}^{-1}$ if our interpretation of the apparent periodicity in the velocity jitter of two stars was interpreted correctly as due to the rotational period. These very high levels of line broadening seemed to rule out macroturbulence. We also discounted transfer of core rotational angular momentum to the surface of the RGB stars because we should have seen significant increases in line broadening where internal mixing manifests itself by changes in atmospheric abundances. Having ruled out alternative explanations, we speculated that a "spin up" in outer envelope rotational velocities could have been caused by the absorption of giant planets. This would imply that metal-poor stars might have such planets and that they exist at larger orbital distances from the host stars than have been found to date in many metal-rich disk stars.

We also observed significant line broadening in the RHB stars. To first order, this makes sense. If the much larger stars near the tip of the RGB stars rotate, then so should their descendents. But perhaps their descendents should be stars whose enhanced rotation helps them shed their outer envelopes, which should lead to core helium-burning stars blueward of the instability strip, known as blue horizontal branch (BHB) stars. Soker (1998) and Siess \& Livio (1999) argued that absorption of a planet by a luminous red giant could explain the high rotation velocities often found among field BHB stars (Kinman et al. 2000; Behr 2003) and cluster BHB stars (Peterson et al. 1983; Peterson 1985a; Peterson et al. 1995; Cohen \& McCarthy 1997; Behr et al. 2000a,b; Recio-Blanco et al. 2002). More generally, theorists have struggled to explain the presence of both RHB and BHB stars in clusters whose stars share the same metallicity. Some "second parameter" must be at work, presumably leading to either larger (RHB) envelope masses or smaller ones (BHB stars). If rotation is the second parameter, then why do RHB stars in the field appear to have rotational velocities

\footnotetext{
${ }^{1} \mathrm{C} 2003$ referred to line broadening as rotational velocities since the values were determined using rotationally-broadened synthetic spectra. Macroturbulence also contributes to line broadening, so throughout this paper we refer to the more general term, line broadening, and refer to $V_{\text {broad }}$ rather than $V_{\text {rot }} \sin i$.
} 
comparable to BHB stars, when allowance is made for their different radii?

C2003 suggested four follow-up studies. First, expand the sample to ascertain if our original sample was unusual in some manner. This paper reports on the results of a "hasty" study of 45 additional metal-poor field stars2. We also sought to obtain line broadening measures for giants in globular clusters, and initial data for four clusters are in hand. Results will be published later. If planets do exist around metal-poor stars, this would suggest that disk instability is a viable mechanism for planet formation, and a high-precision radial velocity survey of roughly two hundred metal-poor field stars was begun (see Sozzetti et al. 2006). Finally, we have to ask if the line broadening is due to rotation or to macroturbulence. This can be determined with very high-resolution, high-S/N spectra that enable Fourier transform studies of line profiles, following Gray (1982; 1984) and Gray \& Toner (1986; 1987). We have completed acquisition and analysis of such spectra and will report on the results in a future paper (Carney et al. 2007).

\section{SELECTION OF STARS FOR STUDY}

C2003 discussed in detail the criteria by which they assembled their list of RGB and RHB field stars for study. These followed mostly the earlier study of Carney \& Latham (1986), which in turn relied on the kinematically-unbiased metal-poor $([\mathrm{Fe} / \mathrm{H}] \leq-1.5)$ samples identified by Bond (1980). He identifield weak-lined stars using objective prism spectroscopy as well as follow-up uvby photometry. Norris, Bessell, \& Pickles (1985) undertook a large program of additional DDO and $R I_{\mathrm{C}}$ photometry. Most of the stars in the C2003 sample had been classified as metal-poor red giants by Anthony-Twarog \& Twarog (1994; hereafter ATT), who supplied estimated reddening and $M_{\mathrm{V}}$ values. All but one of the 45 stars discussed in this paper come from ATT, and most of them were identified originally by Bond (1980). The stars in this study represent most of the stars in ATT with $[\mathrm{Fe} / \mathrm{H}] \leq-1.5$ not studied by $\mathrm{C} 2003$ but within reach of the telescopes employed in that program. To make certain that the stars are all luminous, we have employed the Hipparcos database, which includes 44 of the stars (only BD+25 3410 lacks a measured trigonometric parallax). The parallaxes are all very small and consistent with high luminosities.

\footnotetext{
${ }^{2}$ By "hasty", we mean that some stars were observed for less than two years.
} 


\section{OBSERVATIONS}

The spectra of our new program stars have been obtained in the same fashion as those studied by C2003, using the Center for Astrophysics Digital Speedometers (Latham 1985, 1992), primarily with the 1.5-m Wyeth reflector at the Oak Ridge Observatory in Harvard, Massachusett: 3 , as well as the 1.5-m Tillinghast reflector and the MMT instruments atop Mt. Hopkins in Arizona. The Tillinghast reflector was especially important for the stars south of $-20^{\circ}$ and north of $+62^{\circ}$ in declination. Also as before, the wavelength coverage is $45 \AA$, centered near $5187 \AA$, with a resolution of $8.5 \mathrm{~km} \mathrm{~s}^{-1}$. The signal-to-noise ratio varied from 10 to 50 per resolution element, with a typical value of about 15 .

C2003 described in detail the measurement of the radial velocities. A grid of model atmospheres, defined by $T_{\text {eff }}, \log g$, and $[\mathrm{Fe} / \mathrm{H}]$ values, was computed. The grid spacing in temperature was $250 \mathrm{~K}, 0.5 \mathrm{dex}$ in $\log g$, and 0.5 dex in metallicity for $[\mathrm{Fe} / \mathrm{H}] \leq-1.0$. functions and elemental abundances in which all the " $\alpha$ " elements (O, Ne, Mg, Si, S, Ca, and $\mathrm{Ti}$ ) were enhanced by 0.4 dex relative to the solar abundances. More details may be found in Nördstrom et al. (1994) and C2003.

The program SYNTHE was used to compute synthetic spectra in the wavelength range 5146-5229 A. In the case of the Sun, we obtained an excellent line-by-line match between the Kurucz solar model and synthetic spectrum compared with the observed solar flux spectrum (Kurucz et al. 1984). SYNTHE computes specific intensity at 17 different emergent angles across the stellar disk, and integration over the disk, including the effects of stellar rotation, yields the synthetic flux spectrum. SYNTHE enables us to include the effects of instrumental resolution, which we chose to be a Gaussian with a FWHM of $8.5 \mathrm{~km} \mathrm{~s}^{-1}$, which is appropriate to the instrumentation we employed. The adopted microturbulent velocities were $2 \mathrm{~km}$ $\mathrm{s}^{-1}$, and macroturbulent velocities were $3 \mathrm{~km} \mathrm{~s}^{-1}$. At each combination of temperature, gravity, and metallicity in our grid we computed synthetic spectra employing a wide range of rotational broadening profiles, with $V_{\text {rot }}=0,1,2,4,6,8,10,12,16,20,25,30,35,40$, $50,60,70,80,90,100,110,120$, and $140 \mathrm{~km} \mathrm{~s}^{-1}$.

Once the stellar parameters, $T_{\text {eff }}, \log g$, and $[\mathrm{Fe} / \mathrm{H}]$ had been estimated, we relied on the model atmosphere grid point closest in these variables, paying special attention to the primary variable, temperature. In the case of more than one close match, we employed the template that gave the highest value for the peak correlation, averaged over all the observed spectra.

\footnotetext{
${ }^{3}$ Alas, the Oak Ridge Observatory is now closed, inhibiting such long-term radial velocity work.
} 


\section{STELLAR PARAMETERS}

\subsection{Basic Procedure}

Estimation of stellar atmospheric parameters follows the procedures described by C2003. We make use of photometric methods to compute effective temperatures and bolometric corrections, and we require, therefore, good estimates of the interstellar extinction. We adopted the $M_{\mathrm{V}}$ and $\mathrm{E}(b-y)$ values directly from ATT, except for HD 6833, for which ATT did not derive such parameters, and HDE 232078, which ATT did not study. Both stars lie at low Galactic latitude $(b=-8.0$ and -2.3 , respectively), and are quite distant, so reddening is a special problem.

Table 1 summarizes the photometry employed in our work. The $R-I$ photometry is on the Cousins system. Thanks to the 2MASS Point Source Catalog (Skrutskie et al. 2006)4, we were able to find $V-K$ photometry for all of our new program stars, although we transformed the 2MASS $K$ magnitudes to the "CIT" system following the prescription given in the Explanatory Supplement to the 2MASS Second Incremental Data Release, followed by a transformation to the "TCS" systems as described by Alonso et al. (1994).

Temperature estimates were obtained using the color- $T_{\text {eff }}$ relations derived by Alonso, Arribas, \& Martinez Roger (1999, 2001), based on Infrared Flux Method determinations. The published relations employ the "TCS" system $V-K$ colors, and Johnson $R-I$ colors, so we transformed the $(R-I)_{\mathrm{C}}$ values of Table 1 into the Johnson system, using the relations given by Fernie (1983). When more than one temperature estimate was available, as was most often the case, we employed a simple mean. The average rms scatter when three or more temperatures estimates are available is $45 \mathrm{~K}$.

We assumed stellar masses of $0.8 M_{\odot}$, appropriate for stars at the main sequence turn-off in globular clusters. Calculation of gravities then followed from the $M_{V}$ values, transformed to $M_{\text {bol }}$ after addition of the bolometric correction (Alonso et al. 1999). Table 2 summarizes the resultant atmospheric parameters for our program stars.

\footnotetext{
${ }^{4}$ The 2MASS photometry discussed here were produced by the Two Micron All Sky Survey, which was a joint project of the University of Massachusetts and the Infrared Processing and Analysis Center/California Institute of Technology, funded by the National Aeronautics and Space Administration and the National Science Foundation.
} 


\subsection{Special Cases}

To estimate the interstellar extinction to HD 6833, we exploited the spectroscopic study by Fulbright $(2000)$, who derived $T_{\text {eff }}=4450 \mathrm{~K}, \log g=1.4$, and $[\mathrm{Fe} / \mathrm{H}]=-1.04$. The star has $V=6.75$ and $B-V=1.17$, and its metallicity is a near-perfect match for that of NGC $6723([\mathrm{Fe} / \mathrm{H}]=-1.03$ Kraft \& Ivans 2003). To obtain the same effective temperature from the Alonso et al. (1999) calibrations requires $\mathrm{E}(B-V)=0.11[\mathrm{E}(b-y)=0.076]$, so $(B-V)_{0}=1.06$. The available color-magnitude diagram of the cluster reveals that $\mathrm{M}_{\mathrm{V}} \approx-0.4$ at that color, which we adopt. Note that as a result, our photometric estimate of $\log g$ is 1.6, quite similar to that obtained by Fulbright (2000), who derived a value of 1.4.

In the case of HDE 232078, we relied on the analysis by Burris et al. (2000). They determined the temperature and metallicity using spectroscopic methods, but employed photometry to estimate the gravity, finding $T_{\text {eff }}=4000, \log g=+0.3$, and $[\mathrm{Fe} / \mathrm{H}]=-1.54$. These values enable us to determine the interstellar extinction, under the assumption that the Alonso et al. (1999) color-temperature calibration should yield the same temperature if we have estimated the reddening correctly. The globular cluster M3 has nearly identical metallicity $([\mathrm{Fe} / \mathrm{H}]=-1.47$; Kraft et al. 1992$)$, and a very low reddening $[\mathrm{E}(B-V)=0.01$ $\mathrm{mag}$ ], so in principle we only need to use the Alonso et al. (1999) relations between $V-K$ and $T_{\text {eff }}$ to estimate temperatures of its red giants. Then we simply identify $(V-K)_{0}$ for stars with the same effective temperature as HDE 232078. Using available unpublished M3 $B V$ photometry and 2MASS $K$ magnitudes, we obtain $\mathrm{E}(B-V)=0.56$ mag for HDE 232078 , as well as $M_{\mathrm{V}}=-2.15$. Our photometric estimate for the gravity is $\log g=+0.6$, somewhat higher than that estimated by Burris et al. (2000).

\subsection{Tests of Distance, Gravity, \& $[\mathrm{Fe} / \mathrm{H}]$ Estimations}

We rely on the photometric estimation of $M_{\mathrm{V}}$ values and bolometric corrections to estimate the gravities of our program stars, so we must ask how accurate such estimates are, especially given the relatively large distances to our program stars. We have two methods available: trigonometric parallaxes from HIPPARCOS, and spectroscopic gravities derived during the course of metallicity determinations.

Figure 1 plots $M_{\mathrm{V}}$ values estimated by ATT and, in the special cases here and in C2003, by us, against those derived directly from trigonometric parallaxes. The $1 \sigma$ error bars are almost all very large, since typical parallaxes are only one to two milli-arcseconds, and the uncertainties are often comparable to or even larger than the parallaxes, so that the formal error encompasses physically unrealistic negative parallaxes. We have not plotted the 16 
stars with negative parallaxes, nor the 9 stars whose resultant $M_{\mathrm{V}}$ values are brigher than -4.0 mag since those stars are more luminous than the red giant branch tip stars in globular clusters. All 9 stars have very large errors in $M_{\mathrm{V}}$, the smallest value being 4.85 mag. For the remaining stars whose error bars result in negative parallaxes, we have applied very large error bars so that they run off the left edge of Figure 1. We acknowledge that the Figure is not particularly helpful, but does merit some scrutiny. For example, there are a number of stars for which the parallaxes predict much fainter absolute magnitudes and, therefore, much higher gravities. We have identified three such stars in particular: BD+3 $2782\left(M_{\mathrm{V} \text {,adopted }}\right.$ $\left.=-1.3 ; M_{\mathrm{V}, \pi}=+2.68_{-0.96}^{+0.66}\right) ; \operatorname{HD} 85773\left(M_{\mathrm{V}, \text { adopted }}=-1.97 ; M_{\mathrm{V}, \pi}=+2.41_{-0.84}^{+0.60}\right) ;$ and HD $184711\left(M_{\mathrm{V} \text {,adopted }}=-2.35 ; M_{\mathrm{V}, \pi}=+0.20_{-1.00}^{+0.68}\right)$. For these three stars, Gratton et al. (1996) employed ionization balance to estimate gravities from high-resolution, high-S/N spectra. Our photometry-based gravities for these three stars are, respectively, $\log g=+1.3$, +0.90 , and +0.59 , consistent with high luminosity. Gratton et al. (1996) obtained +1.28 , +0.48 , and +0.15 , even lower gravities, implying even higher luminosities for HD 85773 and HD 184711 than we have estimated. We believe the few disagreements between our derived $M_{\mathrm{V}}$ values and those obtained from parallaxes reflect the uncertainties at the limit of the Hipparcos mission capabilities.

Figure 2 continues the comparisons of spectroscopic and photometric gravities, relying on Gratton et al. (1996) as well as Fulbright (2000). The Figure reveals that the gravities derived by Gratton et al. (1996) are lower than the photometric estimates for almost all of the lowest gravity, highest luminosity stars. Agreement with Fulbright (2000), however, is much better. A least squares fit results in a slope of almost unity (0.92) and modest offset (0.04 dex), and, in particular, a small scatter $( \pm 0.27$ dex). That level of scatter is very satisfying given the difficulties of estimating $M_{\mathrm{V}}$ and, similarly, in determining spectroscopic gravities.

Figure 3 shows how our adopted $[\mathrm{Fe} / \mathrm{H}]$ values, based primarily on the Strömgren photometry calibrations from ATT, compare with results obtained from classical fine analyses of high-S/N, high-resolution spectra of Gratton et al. (1996) and Fulbright (2002). The mean offset in $[\mathrm{Fe} / \mathrm{H}]$ between our adopted values and the 26 stars in common with Gratton et al. (1996) for our entire survey (Carney et al. 2003 and this paper) is $+0.03 \pm 0.04$ dex, with $\sigma=0.22$ dex, in the sense our results minus those of Gratton et al. For Fulbright (2000), the offset for 23 stars in common is $+0.12 \pm 0.04$ dex, $\sigma=0.20$ dex. Both of these results are comparable to the mean difference between the 12 stars in our program that are common to both Gratton et al. (1996) and Fulbright (2000): $<[\mathrm{Fe} / \mathrm{H}]_{\text {Gratton }}-[\mathrm{Fe} / \mathrm{H}]_{\text {Fulbright }}>=$ $+0.14 \pm 0.06, \sigma=0.19$ dex.

Figures 1 through 3 give us confidence that our gravity, luminosity, and metallicity 
estimations are, in general, quite good. Figure 4 plots the derived temperatures and gravities for our program stars vs. the 14 Gyr model isochrones of Straniero \& Chieffi (1991). The two panels show the stars discussed in this paper, and the combined results from this paper plus those stars studied by C2003. There is excellent agreement between the data points and the isochrones on the RGB, suggesting again that our derived atmospheric parameters are quite plausible.

The reader may have noted (as did the referee) that the match to the isochrones in Figure 4 is not perfect, with stars whose gravities are only slightly lower than those of horizontal branch hovering nearer the hotter, more metal-poor isochrones than are most of the lowest-gravity stars. There are several potential contributing factors at work. For one, the two sets of stars have slightly different metallicities. The 25 stars with $\log g \leq 1.2$ have $<[\mathrm{Fe} / \mathrm{H}]>=-1.86 \pm 0.06(\sigma=0.31)$, while the 35 stars with $1.6 \leq \log g \leq 2.3$ have $<[\mathrm{Fe} / \mathrm{H}]>$ $=-2.14 \pm 0.10(\sigma=0.57)$. So a modest shift to more metal-poor isochrones is expected. But there are subtle systematic effects that may also contribute. For example, the lower gravity stars are more luminous and intrinsically rarer, and, hence, more distant. Reddenings may be more susceptible to systematic errors. And the color-temperature transformations may not be as well calibrated in one gravity domain compared to another. We judge the overall agreement between our estimated gravities and temperatures compared to the model isochrones to satisfy the basic test that, on balance, we have reliable stellar atmospheric parameters for our stars.

\subsection{Selection of Synthetic Spectrum Templates}

We used these derived atmospheric parameters to select the optimum synthetic spectrum to derive both the radial velocities and the line broadening. The average correlation value was computed for all the observed spectra of each star using all available rotationally-broadened templates with the adopted values of temperature, gravity and metallicity. The template with rotational broadening that yielded the highest average correlation was chosen for final use in all the radial velocity measures. The final column of Table 2 includes $T_{\text {eff }}, \log g$, $[\mathrm{Fe} / \mathrm{H}]$, and $V_{\text {broad }}$ for the synthetic spectrum employed. 


\section{RADIAL VELOCITIES}

Table 3 provides the individual heliocentric radial velocity results derived for each star using the tool rvsao (Kurtz \& Mink 1998) running inside the IRAF5 environment. The electronic version of the table includes all the radial velocities from C2003 as well as those obtained from this survey. We do this primarily for the convenience of the reader, and to assure ourselves that all the data have been treated consistently. The results quoted in Table 3 reflect only the internal errors. The reader should also be aware that the velocities are on the "native CfA" system defined by our grid of synthetic spectra and nightly observations of the dawn and dusk sky. To transfer these values to an absolute system defined by observations of minor planets, $0.139 \mathrm{~km} \mathrm{~s}^{-1}$ should be added to these values (Stefanik 1999). Gravitational redshifts have also been neglected.

Table 4 summarizes the new results for the 11 stars from C2003 which we have continued to observe, while Table 5 does the same for the new program stars. In both cases, we list the number of observations, the span of the observations in days, the mean radial velocity, and the uncertainty of the mean velocity. Note that for the binary stars, the mean radial velocity is not as appropriate as the systemic velocity that emerges from the orbital solution. For stars with orbital solutions, we therefore list here the systemic velocity and its uncertainty. We also list the measured rms external error, E, and I, the mean of the internal errors, $\sigma_{\text {int }}$, of the velocity measurements (see Kurtz \& Mink 1998). We also show the ratio, E/I, since large values of $\mathrm{E} / \mathrm{I}(>1.5)$ are suggestive of radial-velocity variability.

As discussed by C2003, another powerful indicator of radial velocity variability is the probability, $\mathrm{P}\left(\chi^{2}\right)$, that the $\chi^{2}$ value could be larger than observed due to Gaussian errors for a star that actually has constant velocity. We employ the internal error estimate, $\sigma_{\text {int }}$, for each of $n$ exposures when calculating $\chi^{2}$ :

$$
\chi^{2}=\sum_{i=1}^{n}\left(\frac{x_{i}-<x>}{\sigma_{i, \mathrm{int}}}\right)^{2} .
$$

The values of $\sigma_{\mathrm{i}, \text { int }}$ are computed by rvsao. If these internal error estimates are correct and the actual errors are Gaussian, then the distribution of $\mathrm{P}\left(\chi^{2}\right)$ should be constant from 0 to 1 , except for the velocity variables occupying the region near zero probability. However, we found that use of the internal error estimates led to a distribution of $\mathrm{P}\left(\chi^{2}\right)$ values that was not flat for $\mathrm{P}\left(\chi^{2}\right)>0.05$. Since a flat distribution is expected, we found it necessary to add

\footnotetext{
${ }^{5}$ IRAF (Image Reduction and Analysis Facility) is distributed by the National Optical Astronomy Observatories, which are operated by the Association of Universities for Research in Astronomy, Inc., under contract with the National Science Foundation.
} 
in quadrature a "floor error" of $0.25 \mathrm{~km} \mathrm{~s}^{-1}$ to each internal error estimate. This floor error compensates for various systematic errors, such as shortcomings in the run-to-run zero-point corrections, which are not included in the internal error estimates. The $\mathrm{P}\left(\chi^{2}\right)$ values, derived with the additional "floor error", are listed in Tables 4 and 5. All stars which we have found to be binaries had $\mathrm{P}\left(\chi^{2}\right)<10^{-6}$.

\section{VELOCITY VARIABLES}

\subsection{Identification of Velocity Variables}

Figure 5 shows the distribution of $\mathrm{P}\left(\chi^{2}\right)$ values for 135 of the 138 stars in $\mathrm{C} 2003$ and this paper. For stars with spectroscopic binary orbital solutions, we use the values derived by C2003 from the orbital solutions rather than the values based on the velocity data alone. We have not plotted here the three stars that are clearly binary systems but which do not yet have orbital solutions, HD 4306, BD-1 2582 and HD 108317. As expected, the probability distribution is flat, except for an excess at the lowest values, which we magnify in the bottom two panels. The shaded areas reveal stars known to be spectroscopic binaries, while the unshaded region in the lowest bin shows the stars that are mostly, if not entirely, velocity variables and whose explanation must involve pulsation or velocity jitter.

\subsection{Stars Studied Previously}

We begin with the data obtained for the 11 stars discussed already by C2003 for which we have obtained additional observations. BD+3 2782 shows no sign of any velocity variability, as before.

Three stars, HD 97, HD 3179, and HD 213467, continue to have low $\mathrm{P}\left(\chi^{2}\right)$ values, but other than one or two deviant velocities, appear to have stable velocities, and it is therefore hard to ascribe the apparent variability as due to either orbital motion or velocity jitter.

Four stars are spectroscopic binaries with orbital solutions presented in C2003 (HD 6755, HD 27928, HD+18 2796, and HD+1 3070). None of the orbital solutions are affected significantly, except that the period uncertainties for HD 6755, BD+18 2796, and BD+1 3070 improve from \pm 24 days to \pm 4.6 days, from \pm 71 days to \pm 54 days, and from \pm 12 days to \pm 7 days, respectively.

The two recognized spectroscopic binaries whose orbital solutions could not be determined by C2003, BD-1 2582 and HD 108317, remain resistant to solution. Figure 6 shows 
the current status of our observations. BD-1 2582 now has 52 velocities spanning 7938 days (21.7 years), compared to the results presented in C2003 (37 velocities over 6562 days), while HD 108317 now has time coverage increased from 3432 days to 4573 days (12.5 years) and the number of velocities has increased from 42 to 48 .

HD 121261 remains hard to classify. We have increased the time coverage from 5142 days to 7684 days (21.0 years), and the number of velocities from 12 to 15 . Unfortunately, there is still no suggestion of a periodicity, and the velocity scatter remains modest $(0.85 \mathrm{~km}$ $\left.\mathrm{s}^{-1}\right)$. The star is judged to be relatively luminous, $M_{\mathrm{V}} \approx-1.5$, so velocity jitter could also be responsible.

\subsection{New Velocity Variables}

Following Figure 5, we discuss only those stars in our new sample with $\mathrm{P}\left(\chi^{2}\right)$ values lower than 0.001. Figure [7 shows the velocity histories for all six stars.

HD 4306 appears to be a spectroscopic binary with an eccentric orbit. HD 184711 is a less compelling case for being a spectroscopic binary, but more observations are needed to confirm this conjecture. The star is very luminous $\left(M_{\mathrm{V}} \approx-2.3\right)$, so the variability could as easily be ascribed to velocity jitter.

HD 6833, HD 29574, BD+1 2916, HD 165195, and HDE 232078 all have good velocity coverage and 14 or more measured velocities, and all have very low $\mathrm{P}\left(\chi^{2}\right)$ values $\left(<10^{-6}\right)$. None show any signs of periodicity in their velocities. All of them, except HD 6833, are judged to be luminous, with $M_{\mathrm{V}} \leq-1.7$, so velocity jitter seems to be the best explanation.

\subsection{Velocity Jitter Revisited}

Figure 8 is an update of Figure 8 in C2003, with the stars from Table 5 added. Binary stars are shown as triangles; non-binary stars as circles. All stars with $\mathrm{P}\left(\chi^{2}\right) \leq 0.001$ are plotted as filled triangles or circles. We have not included HD 4306 because it is uncertain

if the very low $\mathrm{P}\left(\chi^{2}\right)$ value is due to its orbital motion, jitter, or both. Its low luminosity $\left(M_{\mathrm{V}} \approx+0.2\right)$ and its velocity vs. time behavior (Figure 7 ) suggest that only orbital motion is at work, but until we have a reliable orbital solution, we cannot say for certain.

The most striking feature of Figure 8 is that the lowest $\mathrm{P}\left(\chi^{2}\right)$ values, $\leq 10^{-6}$, are restricted to the most luminous stars, those with $M_{\mathrm{V}} \leq-1.4$. The second striking feature is that not all stars with such luminosities manifest velocity jitter. We have included binary 
stars with orbital solutions, and their $\mathrm{P}\left(\chi^{2}\right)$ were recomputed following application of the orbital solution (see the discussion in C2003). Thus the $\mathrm{P}\left(\chi^{2}\right)$ values are a measure of nonorbital sources of velocity variability. We find that only 3 of the 14 stars with estimated $-2<M_{\mathrm{V}} \leq-1.4$ show $\mathrm{P}\left(\chi^{2}\right)$ values of less than $10^{-6}$. But all seven of the stars with estimated $M_{\mathrm{V}}$ values of less than -2.0 do show jitter. Our estimated $M_{\mathrm{V}}$ values do not have sufficient precision to ascertain if velocity jitter "turns on" at a particular luminosity or gravity, but that is a reasonable speculation.

Finally, we note the remaining challenge presented by the three lower luminosity/higher gravity stars with $\mathrm{P}\left(\chi^{2}\right)<10^{-3}$. As noted previously, HD 97 and HD $213467\left(M_{\mathrm{V}}=+1.4\right.$ and +1.7 , respectively) may have low $\mathrm{P}\left(\chi^{2}\right)$ values due to one or two poor radial velocity measures. HD 6833, however, does appear to be a legitimate low-luminosity star with variable velocity that has not, as yet, been coupled with orbital motion. Our estimated gravity, $\log g$ $=1.63$, agrees well with that obtained spectroscopically by Fulbright (2006), $\log g=1.4$. Perhaps additional radial velocity monitoring would reveal the star to be a binary.

\section{LINE BROADENING}

\section{1. $\quad$ Measuring Line Broadening}

We have estimated the line broadening, $V_{\text {broad, }}$ following the procedures described by C2003. For each star we selected the synthetic spectrum for use as a template that best matches the derived temperature, gravity, and metallicity parameters, and then chose the rotational broadening value which produced the highest average correlation value. We interpolated the derived correlation values for the two velocities adjacent to the preferred value using a quadratic fit. The derived $V_{\text {broad }}$ values are listed in the final column of Tables 4 and 5. The typical standard error in these values derived from the individual spectra ranged from 0.5 to $2.0 \mathrm{~km} \mathrm{~s}^{-1}$, so the mean values are well determined, at least internally. The systematic effects were also discussed by C2003. Basically, changes of $250 \mathrm{~K}$ in temperature, 0.5 dex in gravity, or 0.5 dex in metallicity, individually led to velocity sensitivities of 3.0, 3.2 , and $3.3 \mathrm{~km} \mathrm{~s}^{-1}$, independent of the derived line broadening. The synthetic spectra were all computed using a macroturbulent velocity of $3 \mathrm{~km} \mathrm{~s}^{-1}$, but low gravity stars may have higher turbulent velocities (see Gray 1982; Gray \& Pallavicini 1989). The line broadening values for more luminous stars would be expected to be affected more strongly by macroturbulence at the higher luminosities. Values that are well below our instrumental resolution are more vulnerable to these uncertain systematic effects.

The referee has inquired about observing conditions when the star underfills the spectro- 
graph's slit, which may lead to a higher instrumental resolving power than we have assumed. For spectra obtained in Massachusetts, (identified by "W" in the detailed velocity summary), the seeing always overfilled the slit by a factor of two or more. In Arizona (identified by "T" or "M"), there were occasional times when the slit was underfilled. We compared line broadening results for those stars with spectra obtained in both Arizona and Massachusetts and found no discernible differences.

\subsection{Comparisons with Other Studies}

The first question we ask is whether our results are in reasonable agreement with other studies, especially those obtained using higher spectral resolution and higher $\mathrm{S} / \mathrm{N}$. We have made comparisons with three such studies.

We have fifteen stars in common with Behr (2003), with a span in $V_{\text {broad }}$ from about 3 to $21 \mathrm{~km} \mathrm{~s}^{-1}$. Behr's observations included higher resolving power, 60,000 (about $5.0 \mathrm{~km}$ $\mathrm{s}^{-1}$ ), higher $\mathrm{S} / \mathrm{N}$ (for the stars in common, his cumulative $\mathrm{S} / \mathrm{N}$ for the stars ranged from 40 to 200 , with 60 being a typical value), and greater wavelength coverage (1000 to $1500 \AA$ ). Nonetheless, despite our limited wavelength coverage, lower resolving power, and lower S/N observations, a linear least squares bissector analysis results in

$$
V_{\text {broad,CfA }}=1.10 \times V_{\text {broad,Behr }}+0.00 \text {. }
$$

The scatter about this relation is only $1.2 \mathrm{~km} \mathrm{~s}^{-1}$. Taking the 15 stars in a star-to-star comparison shows a mean difference in the values of only $0.6 \mathrm{~km} \mathrm{~s}^{-1}$, again, a scatter of 1.2 $\mathrm{km} \mathrm{s}^{-1}$. (The sense is CfA results minus those of Behr 2003.) If we restrict the comparison to only the 7 stars for which our results suggest $V_{\text {rot }} \sin i \leq 6 \mathrm{~km} \mathrm{~s}^{-1}$, the mean offset is only $0.3 \mathrm{~km} \mathrm{~s}^{-1}$, with $\sigma=1.1 \mathrm{~km} \mathrm{~s}^{-1}$.

We compare the results from this paper and from C2003 with the sample of 20 stars for which we obtained spectra with very high resolving power $\left(150,000\right.$, or $\left.2.0 \mathrm{~km} \mathrm{~s}^{-1}\right)$ and very high S/N (generally over 200) using the Gecko spectrograph on the Canada-France-Hawaii Telescope. Details may be found in another paper (Carney et al. 2007). These spectra were analyzed by Fourier transform methods (see Gray 1982), which result in $V_{\text {rot }} \sin i$ and $\zeta_{\mathrm{RT}}$, a measure of the star's macroturbulence dispersion. To derive a "combined" measure of line broadening, $V_{\text {broad }}$, we treat both values as Gaussians, which is not strictly correct, since the macroturbulent dispersion, $\zeta_{\mathrm{RT}}$, does not behave as a Gaussian. Nonetheless, we define

$$
V_{\text {broad,CFHT }}=\left[\left(V_{\text {rot }} \sin i\right)^{2}+f \zeta_{\mathrm{RT}}^{2}\right]^{1 / 2} .
$$

This formulation is due to Massarotti et al. (2007), and as in their study, our value of $f$ was determined empirically, such that the mean offset between the $V_{\text {broad,CfA }}$ and $V_{\text {broad,CFHT }}$ 
values was minimized. We found the best agreement when $f$ is 0.95 , at least for the current sample of metal-poor giants. For the seven stars with $V_{\text {broad,CfA }}<8.5 \mathrm{~km} \mathrm{~s}^{-1}$, the mean offset is $0.0 \pm 0.4 \mathrm{~km} \mathrm{~s}^{-1}$, with $\sigma=0.9 \mathrm{~km} \mathrm{~s}^{-1}$. For the eight stars with $V_{\text {broad,CfA }} \geq 8.5 \mathrm{~km}$ $\mathrm{s}^{-1}$, the mean offset is $0.0 \pm 0.5 \mathrm{~km} \mathrm{~s}^{-1}$, with $\sigma=1.4 \mathrm{~km} \mathrm{~s}^{-1}$, and for all fifteen stars, the mean offset is $0.0 \pm 0.3 \mathrm{~km} \mathrm{~s}^{-1}, \sigma=1.1 \mathrm{~km} \mathrm{~s}^{-1}$.

The top panel of Figure 9 compares the results from Behr (2003) and our CFHT program with those of this paper and C2003. For the CFHT sample, we have eliminated the five variable stars since the temperatures and gravities derived from photometry may be less reliable. For three stars, HD 25532, HD 184266, and HD 195636, line broadening was determined by both Behr (2003) and from our CFHT data, and we have drawn lines connecting the two sets of results. Agreement is excellent, even well below our instrumental resolution of $8.5 \mathrm{~km} \mathrm{~s}^{-1}$.

Another recent study is that of de Medeiros et al. (2006), who measured $V_{\text {rot }} \sin i$ (i.e., $\left.V_{\text {broad }}\right)$ values at a typical resolving power of $50,000\left(6.0 \mathrm{~km} \mathrm{~s}^{-1}\right), \mathrm{S} / \mathrm{N} \approx 80$, and wavelength coverage spanning $\lambda \lambda 3500-9200$. Their work had 22 stars in common with C2003, and with the results from the present paper, there are 35 stars in common. The range in "rotational velocities" is more limited, despite the larger sample size. For only three of the stars in common have we obtained a $V_{\text {broad }}$ value larger than our resolving power, and the largest of those is only $11.7 \mathrm{~km} \mathrm{~s}^{-1}$. The simplest comparison is then just that from starto-star matches. The average difference, in the sense of our values minus those obtained by de Medeiros et al. (2006) is $-0.4 \mathrm{~km} \mathrm{~s}^{-1}$, with $\sigma=2.7 \mathrm{~km} \mathrm{~s}^{-1}$. If we compare only the 21 stars for which we estimate $V_{\text {broad }} \leq 6 \mathrm{~km} \mathrm{~s}^{-1}$, the mean difference is $-1.8 \mathrm{~km} \mathrm{~s}^{-1}$, with $\sigma=2.5 \mathrm{~km} \mathrm{~s}^{-1}$. The bottom panel of Figure 9 shows the comparison graphically. As the quantitative comparisons found, the scatter appears to be larger in the bottom panel than in the top panel, although most of these comparisons are for stars whose line broadening values are comparable to or smaller than either our instrumental resolution or that of de Medeiros et al. (2006). We note that de Medeiros et al. (2006) compared their results with those of Behr (2003). Taking straight differences, we find a mean difference, in the sense of de Medeiros et al. (2006) minus Behr (2003), of $+0.4 \pm 1.0 \mathrm{~km} \mathrm{~s}^{-1}$, with $\sigma=2.8 \mathrm{~km} \mathrm{~s}^{-1}$, for the eight stars common to both programs. The scatter somewhat larger than the value of $1.4 \mathrm{~km} \mathrm{~s}^{-1}$ that de Medeiros et al. quoted for comparisons of all the stars common to C2003, Behr (2003), and Peterson (1983).

We conclude that our results are in good agreement with all of the above other studies, although perhaps somewhat better with Behr (2003) than with de Medeiros et al. (2006). Considering that our spectra have much lower resolution, much lower $\mathrm{S} / \mathrm{N}$, and much smaller wavelength coverage, the power of synthetic spectrum templates is apparent. 


\section{ROTATION OR MACROTURBULENCE?}

Figure 10 shows the line broadening for the stars from Table 5 (left), and those stars plus the results from $\mathrm{C} 2003$ (right). The 45 stars for which we have obtained new results behave the same as the 91 stars studied by C2003. Specifically, stars with $M_{\mathrm{V}}<-2$ show systematically higher levels of line broadening than the fainter stars. As noted earlier, C2003 speculated that while some of the cases might be due to tidal locking in a binary system 6 , some of the apparently elevated line broadening might be due to increased rotation due to the absorption of a giant planet that had an orbital separation of about one AU. This idea was also consistent with the generally high levels of line broadening seen in the RHB stars, which are, of course, the direct descendents of some of the RGB tip stars. The significant line broadening of many of the RHB stars in our program is also apparent in Figure 10.

Line broadening may also be due to macroturbulence. C2003 dismissed this as a cause because the levels of macroturbulence necessary to provide a total line broadening of 9 to 10 $\mathrm{km} \mathrm{s}^{-1}$ and higher seemed improbable. Gray (1982) and Gray \& Pallavicini (1989) reported macroturbulent velocities for $\mathrm{K}$ giants (luminosity class III), and all values were smaller than $6.5 \mathrm{~km} \mathrm{~s}^{-1}$. Even considering luminosity classes II-III and II, Gray \& Toner (1986) found macroturbulent velocities to be smaller than $8 \mathrm{~km} \mathrm{~s}^{-1}$. However, these results were derived from studies of metal-rich disk stars. An obvious question is whether lower-mass, older, more metal-poor halo giants have elevated levels of macroturbulence compared to disk giants. Carney et al. (2007) do not, in fact, find significant differences in $\zeta_{\mathrm{RT}}$ between metal-rich disk and metal-poor halo red giants.

What about velocity jitter? Let us consider only the RGB stars and exclude the known binary systems, where tidal interactions may have contributed to the rotation and line broadening. We have noted that velocity jitter begins to appear at only the highest luminosities, $M_{\mathrm{V}} \leq-1.4$, and especially for $M_{\mathrm{V}} \leq-2.0$. These are also the luminosity levels for which line broadening measures are highest. In Figure 11 we replace luminosity with $\log \mathrm{P}\left(\chi^{2}\right)$ and compare directly with $V_{\text {broad. }}$ Open red circles are stars with $-2.0<M_{\mathrm{V}} \leq-1.5$. Filled red circles are stars with $M_{\mathrm{V}} \leq-2.0$. As the Figure shows, some of the luminous RGB stars have normal $\mathrm{P}\left(\chi^{2}\right)$ and modest but typical line broadening. However, a significant number of the most luminous stars have very low values of $\mathrm{P}\left(\chi^{2}\right)$, and they also tend to have much higher than average values of $V_{\text {broad }}$. In other words, it appears that there is a strong correlation between velocity jitter and line broadening. This is not a matter of "velocity smearing" during individual observations. The exposure times are far too short, typically

\footnotetext{
${ }^{6} \mathrm{~A}$ prime example of this is CD-37 14010, with $M_{\mathrm{V}}=-1.9, V_{\mathrm{broad}}=19.4 \mathrm{~km} \mathrm{~s}^{-1}$, and an orbital period of 65.55 days.
} 
a few minutes, compared to the physical timescales for major atmospheric changes in very large red giant stars (with timescales of days to months). This Figure suggests that velocity jitter and line broadening share a common cause.

How do we explain the significant line broadening measured for the RHB stars? Rotation may play a significant role, but we suspect the macroturbulence contributes to the broadening. Gray (1982) first noted the correlation between macroturbulent velocities and effective temperature in giant stars, with $\zeta_{\mathrm{RT}}$ rising from around $4 \mathrm{~km} \mathrm{~s}^{-1}$ for K2 III stars to $7 \mathrm{~km} \mathrm{~s}^{-1}$ for G2 III stars. Gray \& Toner (1986) found similar behavior for bright giants, luminosity classes II and II-III. Figure 12 shows our measured line broadening for RHB stars as a function of $T_{\text {eff }}$. Qualitatively, the trend matches the expected increase in $\zeta_{\mathrm{RT}}$ as a function of $T_{\text {eff }}$. There are quantitative difficulties, however, since the line broadening seen among the RHB stars is much larger than the macroturbulent velocities seen in metal-rich disk giants. Rotation should certainly not be ruled out, either. If the most luminous RGB stars have only very modest levels of rotation, their smaller descendents should have significant rotation. Very high-resolution, high-S/N spectra of such stars will enable us to explore the roles of rotation and macroturbulence in both RGB and RHB stars.

\section{CONCLUSIONS}

We have obtained 349 new radial velocities and line broadening measures for 45 metalpoor RGB and RHB stars, as well as 57 such measures for 11 of the stars we studied previously (C2003). A comparison of our derived values for line broadening with results from Behr (2003) and de Medeiros et al. (2006) shows that our lower-resolution, lower$\mathrm{S} / \mathrm{N}$, and limited wavelength coverage spectra yield excellent results. We believe the good agreement testifies to the power of high-resolution synthetic spectra as templates. We have identified one new spectroscopic binary, HD 4306, and a possible second one, HD 184711, although we note that the latter's radial velocity variability may be due to velocity jitter rather than orbital motion.

We draw attention to the observed correlation between variable radial velocity ("jitter") and line broadening. The significant line broadening seen in the metal-poor field RHB stars is hard to explain: it may be a combination of "spin up" when a slowly rotating luminous RGB star settles on to the horizontal branch, or temperature-dependent macroturbulence may be involved. Very high-resolution and very high-S/N spectroscopy should reveal the relative balance of rotation and macroturbulence in RGB and RHB stars.

We thank R. Davis for his many years of service maintaining the database for the CfA 
digital speedometers, and J. Caruso, J. Zajac, P. Berlind, and G. Torres for making many of the observations. BWC thanks the National Science Foundation for grants AST-9988156 and AST-0305431 to the University of North Carolina. JBL thanks the National Science Foundation for grants AST-9988247 and AST-0307340 to Bowling Green State University. We especially acknowledge the great utility of the 2MASS database, as well as the SIMBAD database, maintained by the CDS in Strasbourg, France. 
Table 1. Photometry of Program Stars

\begin{tabular}{|c|c|c|c|c|c|c|c|c|}
\hline Star & $\mathrm{E}(b-y)$ & $(b-y)$ & $V-R_{J}$ & Ref & $(R-I)_{C}$ & Ref & $V-K$ & Ref \\
\hline HD 2665 & 0.049 & 0.551 & 0.729 & 1 & $\ldots$ & & 2.18 & 6 \\
\hline HD 2796 & 0.005 & 0.542 & 0.718 & 1 & 0.478 & 3 & 2.28 & 9 \\
\hline HD 4306 & 0.020 & 0.531 & 0.711 & 1 & 0.475 & 3 & 2.18 & 7 \\
\hline HD 6268 & 0.012 & 0.599 & $\ldots$ & & $\ldots$ & & 2.42 & 9 \\
\hline HD 6229 & 0.020 & 0.489 & $\ldots$ & & $\cdots$ & & 2.05 & 9 \\
\hline HD 6833 & 0.076 & 0.753 & 0.947 & 1 & $\ldots$ & & 2.94 & 9 \\
\hline HD 8724 & 0.029 & 0.683 & 0.887 & 1 & $\ldots$ & & 2.73 & 9 \\
\hline HD 11582 & 0.005 & 0.464 & $\ldots$ & & 0.431 & 3 & 1.91 & 9 \\
\hline HD 13979 & 0.000 & 0.503 & $\cdots$ & & $\ldots$ & & 2.11 & 9 \\
\hline CD-36 1052 & 0.011 & 0.342 & $\cdots$ & & 0.320 & 3 & 1.46 & 9 \\
\hline HD 21022 & 0.000 & 0.651 & $\cdots$ & & 0.530 & 3 & 2.59 & 9 \\
\hline CD-24 1782 & 0.001 & 0.468 & $\ldots$ & & 0.421 & 3 & 2.03 & 9 \\
\hline HD 25532 & 0.053 & 0.482 & 0.660 & 1 & $\ldots$ & & 1.95 & 7 \\
\hline HD 26297 & 0.001 & 0.739 & 0.925 & 1 & 0.603 & 3 & 2.89 & 7 \\
\hline $\mathrm{BD}+6648$ & 0.088 & 0.875 & 1.066 & 1 & 0.737 & 3 & 3.28 & 9 \\
\hline HD 29574 & 0.036 & 0.036 & 1.159 & 1 & 0.732 & 3 & 3.57 & 9 \\
\hline HD 33771 & 0.015 & 0.591 & $\ldots$ & & 0.512 & 3 & 2.46 & 9 \\
\hline HD 41667 & 0.000 & 0.624 & $\ldots$ & & $\ldots$ & & 2.60 & 9 \\
\hline HD 44007 & 0.067 & 0.560 & 0.754 & 1 & 0.494 & 3 & 2.385 & 7 \\
\hline HD 74462 & 0.017 & 0.665 & 0.850 & 1 & $\ldots$ & & 2.675 & 9 \\
\hline $\mathrm{BD}+541323$ & 0.003 & 0.470 & 0.642 & 1 & $\ldots$ & & 1.965 & 7 \\
\hline $\mathrm{BD}+581218$ & 0.000 & 0.515 & 0.695 & 1 & 0.450 & 4 & 2.175 & 8 \\
\hline HD 85773 & 0.015 & 0.788 & 0.945 & 2 & 0.587 & 2 & 2.91 & 9 \\
\hline HD 88609 & 0.000 & 0.675 & 0.852 & 1 & 0.570 & 4 & 2.59 & 8 \\
\hline HD 101063 & 0.027 & 0.499 & $\ldots$ & & 0.459 & 3 & 2.11 & 9 \\
\hline $\mathrm{BD}+521601$ & 0.000 & 0.555 & 0.723 & 1 & $\ldots$ & & 2.245 & 8 \\
\hline HD 104893 & 0.051 & 0.823 & 1.000 & 2 & 0.643 & 3 & 3.00 & 9 \\
\hline HD 106373 & 0.044 & 0.338 & $\ldots$ & & $\ldots$ & & 1.51 & 8 \\
\hline $\mathrm{BD}+42621$ & 0.003 & 0.595 & 0.764 & 1 & $\ldots$ & & 2.43 & 9 \\
\hline $\mathrm{BD}+12916$ & 0.011 & 0.911 & 1.049 & 1 & 0.685 & 3 & 3.24 & 9 \\
\hline HD 128279 & 0.039 & 0.469 & $\ldots$ & & 0.439 & 3 & 1.99 & 8 \\
\hline HD 151559 & 0.069 & 0.584 & $\ldots$ & & $\ldots$ & & 2.40 & 9 \\
\hline $\mathrm{BD}+173248$ & 0.040 & 0.486 & 0.638 & 1 & $\ldots$ & & 2.04 & 8 \\
\hline $\mathrm{BD}+253410$ & 0.083 & 0.440 & $\ldots$ & & $\ldots$ & & 1.835 & 5 \\
\hline HD 165195 & 0.099 & 0.920 & 1.076 & 1 & 0.710 & 4 & 3.30 & 8 \\
\hline HD 166161 & 0.209 & 0.686 & 0.891 & 1 & 0.622 & 3 & 2.745 & 5 \\
\hline HD 184711 & 0.081 & 0.938 & $\ldots$ & & 0.740 & 3 & 3.31 & 9 \\
\hline HDE 232078 & 0.400 & 1.437 & 1.62 & 1 & $\ldots$ & & 4.50 & 9 \\
\hline
\end{tabular}


Table 1-Continued

\begin{tabular}{crrrrrrrrr}
\hline \hline Star & $\mathrm{E}(b-y)$ & $(b-y)$ & $V-R_{J}$ & Ref & $(R-I)_{C}$ & Ref & $V-K$ & Ref \\
\hline HD 187111 & 0.081 & 0.835 & 1.026 & 1 & 0.678 & 3 & 3.20 & 9 \\
HD 190287 & 0.026 & 0.503 & $\ldots$ & & 0.447 & 3 & 2.13 & 9 \\
HD 204543 & 0.024 & 0.635 & 0.817 & 1 & $\ldots$ & & 2.55 & 9 \\
HD 215601 & 0.009 & 0.532 & $\ldots$ & & 0.472 & 3 & 2.20 & 9 \\
HD 216143 & 0.013 & 0.690 & 0.874 & 1 & $\ldots$ & & 2.72 & 9 \\
HD 218620 & 0.007 & 0.720 & $\ldots$ & & $\ldots$ & & 2.76 & 9 \\
HD 218857 & 0.021 & 0.500 & 0.687 & 1 & $\ldots$ & & 2.12 & 9 \\
\hline
\end{tabular}

References. - (1) Stone 1983; (2) Carney 1980; (3) Norris, Bessell, \& Pickles 1985; (4) Carney 1983; (5) Carney \& Latham 1986; (6) Arribas \& Martinez Roger 1987; (7) Alonso, Arribas, \& Martinez Roger 1994; (8) Alonso, Arribas, \& Martinez Roger 1998; (9) 2MASS. 
Table 2. Stellar Parameters

\begin{tabular}{|c|c|c|c|c|c|c|c|c|}
\hline Star & $\alpha(\mathrm{J} 2000) \delta$ & {$[\mathrm{Fe} / \mathrm{H}]$} & $M_{\mathrm{V}}$ & $T_{\text {eff }}$ & $\log g$ & $R / R_{\odot}$ & $\mathrm{d}(\mathrm{pc})$ & $\begin{array}{c}\text { Synthetic Spectrum } \\
T_{\text {eff }} / \log g /[\mathrm{Fe} / \mathrm{H}] / V_{\text {rot }}\end{array}$ \\
\hline HD 2665 & $003045.4+570353$ & -1.99 & 0.66 & 5000 & 2.34 & 10.1 & 240 & $5000 / 2.5 /-2.0 / 0$ \\
\hline HD 2796 & $003116.9-164740$ & -2.45 & -0.81 & 4830 & 1.67 & 21.8 & 720 & $4750 / 1.5 /-2.5 / 8$ \\
\hline HD 4306 & $004527.1-093239$ & -2.49 & 0.19 & 4940 & 2.12 & 13.0 & 560 & $5000 / 2.0 /-2.5 / 4$ \\
\hline HD 6268 & $010318.1-275250$ & -2.25 & -1.06 & 4710 & 1.51 & 26.2 & 670 & $4750 / 1.5 /-2.0 / 4$ \\
\hline HD 6229 & $010336.4+234606$ & -1.09 & 0.84 & 5160 & 2.49 & 8.4 & 340 & $5250 / 2.5 /-1.0 / 8$ \\
\hline HD 6833 & $010952.2+544420$ & -1.04 & -0.40 & 4450 & 1.63 & 22.8 & 230 & $4500 / 1.5 /-1.0 / 8$ \\
\hline HD 8724 & $012617.5+170735$ & -2.01 & -1.11 & 4480 & 1.36 & 31.1 & 730 & $4500 / 1.5 /-2.0 / 6$ \\
\hline HD 11582 & $015300.2-341736$ & -1.57 & 1.88 & 5150 & 2.90 & 5.3 & 340 & $5000 / 3.0 /-1.5 / 1$ \\
\hline HD 13979 & $021520.8-255454$ & -2.55 & -0.33 & 4980 & 1.93 & 16.1 & 800 & $5000 / 2.0 /-2.5 / 2$ \\
\hline CD-36 1052 & $024737.4-360627$ & -2.19 & 0.62 & 5980 & 2.69 & 6.7 & 720 & $6000 / 2.5 /-2.0 / 16$ \\
\hline HD 21022 & $032221.6-325940$ & -1.17 & -1.17 & 4500 & 1.35 & 31.4 & 1190 & $4500 / 1.5 /-1.0 / 4$ \\
\hline CD-24 1782 & $033841.4-240250$ & -2.66 & 0.61 & 5150 & 2.38 & 9.6 & 730 & $5250 / 2.5 /-2.5 / 1$ \\
\hline HD 25532 & $040411.0+232427$ & -1.33 & 0.79 & 5320 & 2.54 & 8.0 & 270 & $5250 / 2.5 /-1.5 / 8$ \\
\hline HD 26297 & $040903.4-155327$ & -1.67 & -1.48 & 4280 & 1.08 & 42.9 & 620 & $4250 / 1.0 /-1.5 / 6$ \\
\hline $\mathrm{BD}+6648$ & $041313.1+063601$ & -1.82 & -1.79 & 4160 & 0.87 & 54.6 & 1250 & $4250 / 1.0 /-2.0 / 6$ \\
\hline HD 29574 & $043855.7-132048$ & -1.63 & -2.11 & 3960 & 0.57 & 77.3 & 1160 & $4000 / 0.5 /-1.5 / 10$ \\
\hline HD 33771 & $051049.6-374903$ & -1.93 & -0.36 & 4680 & 1.77 & 19.3 & 890 & $4750 / 1.5 /-2.0 / 6$ \\
\hline HD 41667 & $060503.6-325939$ & -1.18 & +0.07 & 4580 & 1.89 & 16.8 & 490 & $4500 / 2.0 /-2.0 / 4$ \\
\hline HD 44007 & $061848.4-145043$ & -1.23 & 1.83 & 4980 & 2.80 & 5.9 & 150 & $5000 / 3.0 /-1.0 / 0$ \\
\hline HD 74462 & $084820.6+672659$ & -1.60 & -0.84 & 4510 & 1.49 & 26.8 & 790 & $4500 / 1.5 /-1.5 / 4$ \\
\hline $\mathrm{BD}+541323$ & $094219.4+532826$ & -1.85 & 0.69 & 5120 & 2.41 & 9.3 & 530 & $5000 / 2.5 /-2.0 / 4$ \\
\hline $\mathrm{BD}+581218$ & $095238.6+575458$ & -2.84 & 0.39 & 4950 & 2.20 & 11.8 & 820 & $5000 / 2.0 /-3.0 / 4$ \\
\hline HD 85773 & $095339.2-225008$ & -2.22 & -1.97 & 4300 & 0.90 & 53.0 & 1820 & $4250 / 1.0 /-2.0 / 6$ \\
\hline HD 88609 & $101428.9+533339$ & -2.58 & -1.42 & 4470 & 1.22 & 36.5 & 1010 & $4500 / 1.0 /-2.5 / 4$ \\
\hline HD 101063 & $113740.4-285104$ & -1.09 & 2.74 & 5070 & 3.21 & 3.7 & 210 & $5000 / 3.0 /-1.0 / 2$ \\
\hline $\mathrm{BD}+521601$ & $115959.0+514617$ & -1.49 & 0.13 & 4800 & 2.03 & 14.3 & 540 & $4750 / 2.0 /-1.5 / 4$ \\
\hline HD 104893 & $120443.1-291105$ & -1.78 & -1.70 & 4270 & 0.99 & 47.6 & 1400 & $4250 / 1.0 /-2.0 / 2$ \\
\hline HD 106373 & $121413.3-281506$ & -2.48 & 0.57 & 6160 & 2.71 & 6.5 & 430 & $6250 / 2.5 /-2.5 / 12$ \\
\hline $\mathrm{BD}+42621$ & $122844.6+040126$ & -2.51 & -0.87 & 4710 & 1.58 & 24.1 & 1460 & $4750 / 1.5 /-2.5 / 2$ \\
\hline $\mathrm{BD}+12916$ & $142145.1+004659$ & -1.45 & -1.76 & 4040 & 0.78 & 60.1 & 1920 & $4000 / 0.5 /-1.5 / 8$ \\
\hline HD 128279 & $143648.5-290646$ & -1.97 & 1.86 & 5290 & 2.94 & 5.0 & 160 & $5250 / 3.0 /-2.0 / 0$ \\
\hline HD 151559 & $164908.1-273415$ & -1.01 & 1.65 & 4970 & 2.73 & 6.4 & 250 & $5000 / 2.5 /-1.0 / 0$ \\
\hline $\mathrm{BD}+173248$ & $172814.4+173035$ & -2.07 & 0.65 & 5240 & 2.44 & 9.0 & 510 & $5250 / 2.5 /-2.0 / 4$ \\
\hline $\mathrm{BD}+253410$ & $180203.2+250041$ & -1.37 & 0.79 & 5740 & 2.69 & 6.7 & 450 & $5750 / 2.5 /-1.5 / 10$ \\
\hline HD 165195 & $180440.0+034644$ & -2.16 & -2.14 & 4200 & 0.76 & 61.9 & 640 & $4250 / 0.5 /-2.0 / 8$ \\
\hline HD 166161 & $180940.6-084645$ & -1.33 & 0.79 & 5070 & 2.43 & 9.0 & 190 & $5000 / 2.5 /-1.5 / 4$ \\
\hline
\end{tabular}


Table 2-Continued

\begin{tabular}{lccccccrc}
\hline \hline \multicolumn{1}{c}{ Star } & \multicolumn{1}{c}{$\alpha(\mathrm{J} 2000) \delta$} & {$[\mathrm{Fe} / \mathrm{H}]$} & \multicolumn{1}{c}{$M_{\mathrm{V}}$} & $T_{\text {eff }}$ & $\log g$ & $R / R_{\odot}$ & $\mathrm{d}(\mathrm{pc})$ & $\begin{array}{c}\text { Synthetic Spectrum } \\
T_{\text {eff }} / \log g /[\mathrm{Fe} / \mathrm{H}] / V_{\text {rot }}\end{array}$ \\
\hline HD 184711 & $193711.9-394437$ & -2.29 & -2.35 & 4090 & 0.59 & 75.4 & 1000 & $4000 / 0.5 /-2.5 / 6$ \\
HDE 232078 & $193812.1+164826$ & -1.54 & -2.15 & 4000 & 0.57 & 76.7 & 660 & $4000 / 0.5 /-1.5 / 10$ \\
HD 187111 & $194839.5-120719$ & -1.65 & -1.54 & 4260 & 1.04 & 44.6 & 610 & $4250 / 1.0 /-1.5 / 4$ \\
HD 190287 & $200538.2-345510$ & -1.09 & 2.63 & 5090 & 3.18 & 3.8 & 150 & $5000 / 3.0 /-1.0 / 2$ \\
HD 204543 & $212928.2-033055$ & -1.85 & -1.09 & 4610 & 1.44 & 28.1 & 720 & $4500 / 1.5 /-2.0 / 6$ \\
HD 215601 & $224648.0-315218$ & -1.56 & -0.17 & 4865 & 1.95 & 15.8 & 530 & $4750 / 2.0 /-1.5 / 4$ \\
HD 216143 & $225031.0-065449$ & -2.01 & -1.42 & 4445 & 1.21 & 36.8 & 690 & $4500 / 1.0 /-2.0 / 6$ \\
HD 218620 & $230940.1-305436$ & -1.55 & -1.22 & 4370 & 1.25 & 35.3 & 1300 & $4250 / 1.0 /-1.5 / 4$ \\
HD 218857 & $231124.6-161504$ & -2.15 & 0.81 & 5040 & 2.42 & 9.2 & 410 & $5000 / 2.5 /-2.0 / 2$ \\
\hline
\end{tabular}


Table 3. Radial Velocities

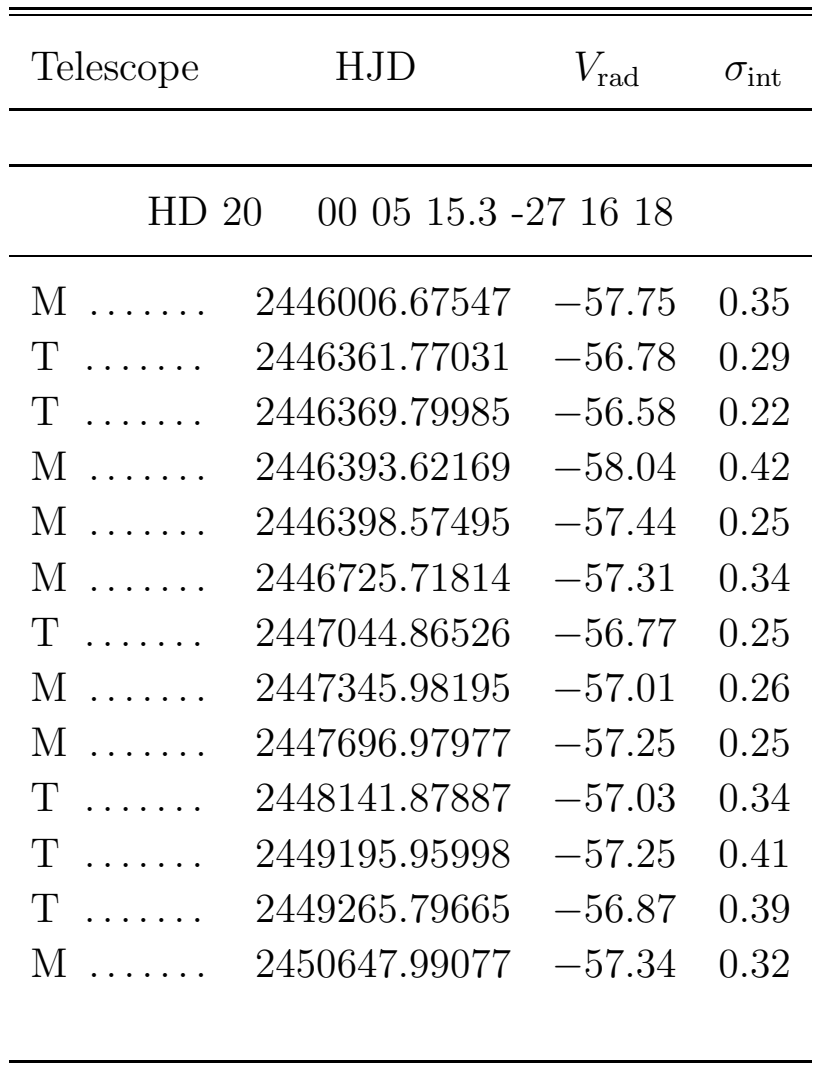


Table 4. Radial Velocity Summary - Prior Study

\begin{tabular}{lrrrrrrrrrr}
\hline \hline \multicolumn{1}{c}{ Star } & N & Span & $\left\langle V_{\text {rad }}\right\rangle$ & $\sigma\left(V_{\text {rad }}\right)$ & $\mathrm{E}$ & $\mathrm{I}$ & $\mathrm{E} / \mathrm{I}$ & $\chi^{2}$ & $\mathrm{P}\left(\chi^{2}\right)$ & $V_{\text {broad }}$ \\
\hline HD 97 & 42 & 7687 & 75.64 & 0.13 & 0.87 & 0.59 & 1.47 & 78.61 & 0.000370 & 3.8 \\
HD 3179 & 20 & 7503 & -74.73 & 0.22 & 0.97 & 0.64 & 1.51 & 37.17 & 0.007559 & 5.5 \\
HD 6755 & 36 & 7713 & -318.36 & 0.12 & 5.96 & 0.42 & 14.17 & 6676.71 & 0.000000 & 3.3 \\
HD 27928 & 38 & 6830 & 44.40 & 0.17 & 2.51 & 0.43 & 5.83 & 1188.63 & 0.000000 & 0.0 \\
BD -1 2582 & 52 & 7938 & 0.35 & 0.19 & 1.34 & 0.68 & 1.97 & 232.78 & 0.000000 & 6.6 \\
HD 108317 & 48 & 4573 & 7.27 & 0.15 & 1.03 & 0.56 & 1.82 & 165.77 & 0.000000 & 5.1 \\
BD+3 2782 & 14 & 7363 & 31.97 & 0.16 & 0.60 & 0.52 & 1.16 & 17.11 & 0.194256 & 5.0 \\
HD 121261 & 15 & 7684 & 100.03 & 0.22 & 0.85 & 0.44 & 1.92 & 61.15 & 0.000000 & 7.7 \\
BD+18 2976 & 106 & 7528 & -167.97 & 0.09 & 3.54 & 0.55 & 6.47 & 4915.69 & 0.000000 & 5.1 \\
BD+1 3070 & 43 & 7731 & -330.11 & 0.11 & 2.86 & 0.66 & 4.34 & 918.75 & 000000 & 4.9 \\
HD 213467 & 14 & 7627 & -72.50 & 0.19 & 0.71 & 0.44 & 1.59 & 42.87 & 0.000047 & 1.8 \\
& & & & & & & & & & \\
\hline
\end{tabular}


Table 5. Radial Velocity Summary - New Program

\begin{tabular}{|c|c|c|c|c|c|c|c|c|c|c|}
\hline Star & $\mathrm{N}$ & Span & $<V_{\text {rad }}>$ & $\sigma\left(V_{\mathrm{rad}}\right)$ & $\mathrm{E}$ & I & $\mathrm{E} / \mathrm{I}$ & $\chi^{2}$ & $\mathrm{P}\left(\chi^{2}\right)$ & $V_{\text {broad }}$ \\
\hline HD 2665 & 7 & 8066 & -382.68 & 0.22 & 0.59 & 0.47 & 1.26 & 15.20 & 0.018728 & 0.5 \\
\hline HD 2796 & 5 & 763 & -60.99 & 0.24 & 0.51 & 0.53 & 0.96 & 2.45 & 0.654283 & 7.0 \\
\hline HD 4306 & 14 & 1242 & -66.48 & 1.38 & 5.17 & 0.53 & 9.75 & 1334.63 & 0.000000 & 0.9 \\
\hline HD 6268 & 6 & 745 & 38.68 & 0.24 & 0.58 & 0.40 & 1.40 & 10.65 & 0.058702 & 4.3 \\
\hline HD 6229 & 6 & 736 & -90.88 & 0.17 & 0.36 & 0.41 & 0.87 & 3.72 & 0.589905 & 7.0 \\
\hline HD 6833 & 34 & 7755 & -243.14 & 0.11 & 0.62 & 0.33 & 1.89 & 116.20 & 0.000000 & 7.4 \\
\hline HD 8724 & 7 & 811 & -113.19 & 0.20 & 0.53 & 0.45 & 1.18 & 9.59 & 0.143010 & 6.0 \\
\hline HD 11582 & 6 & 745 & 21.53 & 0.19 & 0.48 & 0.41 & 1.16 & 7.42 & 0.191459 & 0.5 \\
\hline HD 13979 & 5 & 453 & 51.62 & 0.21 & 0.15 & 0.48 & 0.31 & 0.39 & 0.983112 & 3.7 \\
\hline CD-36 1052 & 5 & 741 & 304.59 & 0.30 & 0.36 & 0.68 & 0.53 & 1.11 & 0.893207 & 14.2 \\
\hline HD 21022 & 5 & 749 & 122.29 & 0.26 & 0.59 & 0.55 & 1.07 & 4.90 & 0.297427 & 5.0 \\
\hline CD-24 1782 & 6 & 741 & 101.43 & 0.26 & 0.63 & 0.50 & 1.25 & 9.00 & 0.109119 & 0.5 \\
\hline HD 25532 & 5 & 560 & -112.24 & 0.20 & 0.39 & 0.44 & 0.89 & 3.27 & 0.513139 & 8.3 \\
\hline HD 26297 & 5 & 419 & 14.83 & 0.25 & 0.56 & 0.43 & 1.31 & 5.59 & 0.231752 & 5.6 \\
\hline $\mathrm{BD}+6648$ & 4 & 403 & -143.46 & 0.20 & 0.27 & 0.41 & 0.65 & 1.30 & 0.728639 & 6.1 \\
\hline HD 29574 & 22 & 7427 & 19.80 & 0.31 & 1.46 & 0.59 & 2.45 & 132.26 & 0.000000 & 10.1 \\
\hline HD 33771 & 4 & 594 & -14.24 & 0.19 & 0.37 & 0.39 & 0.97 & 3.04 & 0.385667 & 5.0 \\
\hline HD 41667 & 3 & 356 & 296.73 & 0.21 & 0.36 & 0.36 & 1.01 & 1.94 & 0.379803 & 5.0 \\
\hline HD 44007 & 5 & 387 & 161.83 & 0.23 & 0.33 & 0.52 & 0.62 & 1.63 & 0.804223 & 0.5 \\
\hline HD 74462 & 7 & 745 & -169.03 & 0.15 & 0.39 & 0.36 & 1.10 & 7.51 & 0.276206 & 4.6 \\
\hline $\mathrm{BD}+541323$ & 5 & 771 & -67.11 & 0.25 & 0.52 & 0.56 & 0.94 & 4.42 & 0.352620 & 3.0 \\
\hline $\mathrm{BD}+581218$ & 5 & 1013 & -304.12 & 0.31 & 0.36 & 0.70 & 0.51 & 1.09 & 0.895896 & 1.5 \\
\hline HD 85773 & 5 & 794 & 147.41 & 0.23 & 0.41 & 0.52 & 0.79 & 1.09 & 0.599917 & 4.2 \\
\hline HD 88609 & 5 & 738 & -38.49 & 0.23 & 0.40 & 0.51 & 0.78 & 2.27 & 0.686534 & 4.0 \\
\hline HD 101063 & 16 & 1036 & 182.34 & 0.21 & 0.86 & 0.52 & 1.64 & 26.72 & 0.031118 & 3.8 \\
\hline $\mathrm{BD}+521601$ & 6 & 1037 & -46.95 & 0.17 & 0.29 & 0.42 & 0.67 & 2.45 & 0.784585 & 4.6 \\
\hline HD 104893 & 8 & 623 & 24.12 & 0.16 & 0.24 & 0.44 & 0.55 & 2.12 & 0.953138 & 3.0 \\
\hline HD 106373 & 7 & 1062 & 83.67 & 0.23 & 0.40 & 0.61 & 0.66 & 2.89 & 0.822853 & 13.7 \\
\hline $\mathrm{BD}+42621$ & 6 & 1011 & -41.96 & 0.26 & 0.30 & 0.63 & 0.47 & 1.32 & 0.932883 & 2.5 \\
\hline $\mathrm{BD}+12916$ & 14 & 1003 & -12.10 & 0.22 & 0.82 & 0.59 & 1.39 & 25.22 & 0.021587 & 8.2 \\
\hline HD 128279 & 7 & 616 & -76.54 & 0.23 & 0.61 & 0.44 & 1.38 & 9.85 & 0.131045 & 0.5 \\
\hline HD 151559 & 4 & 377 & 16.48 & 0.19 & 0.35 & 0.39 & 0.91 & 2.41 & 0.492020 & 0.0 \\
\hline $\mathrm{BD}+173248$ & 6 & 1091 & -146.87 & 0.23 & 0.37 & 0.55 & 0.67 & 2.30 & 0.806028 & 3.8 \\
\hline $\mathrm{BD}+253410$ & 6 & 1095 & -187.76 & 0.32 & 0.67 & 0.77 & 0.87 & 4.77 & 0.444779 & 9.7 \\
\hline HD 165195 & 16 & 7714 & -0.33 & 0.25 & 1.00 & 0.46 & 2.15 & 69.53 & 0.000000 & 7.6 \\
\hline HD 166161 & 6 & 900 & 68.33 & 0.18 & 0.32 & 0.43 & 0.74 & 3.21 & 0.667236 & 4.0 \\
\hline HD 184711 & 6 & 475 & 102.21 & 1.29 & 3.15 & 0.49 & 6.39 & 1236.97 & 0.000000 & 7.7 \\
\hline
\end{tabular}


Table 5-Continued

\begin{tabular}{lrrrrrrrrrr}
\hline \hline \multicolumn{1}{c}{ Star } & $\mathrm{N}$ & $\mathrm{Span}$ & $\left\langle V_{\text {rad }}\right\rangle$ & $\sigma\left(V_{\text {rad }}\right)$ & $\mathrm{E}$ & $\mathrm{I}$ & $\mathrm{E} / \mathrm{I}$ & $\chi^{2}$ & $\mathrm{P}\left(\chi^{2}\right)$ & $V_{\text {broad }}$ \\
\hline HDE 232078 & 19 & 7917 & -387.16 & 0.34 & 1.50 & 0.54 & 2.77 & 168.72 & 0.000000 & 10.9 \\
HD 187111 & 5 & 781 & -186.48 & 0.18 & 0.11 & 0.39 & 0.28 & 0.31 & 0.989185 & 5.2 \\
HD 190287 & 5 & 538 & 143.32 & 0.19 & 0.38 & 0.44 & 0.87 & 3.22 & 0.521124 & 3.4 \\
HD 204543 & 7 & 7570 & -98.35 & 0.17 & 0.37 & 0.45 & 0.83 & 6.86 & 0.334038 & 5.0 \\
HD 215601 & 5 & 749 & -37.14 & 0.21 & 0.48 & 0.38 & 1.25 & 6.73 & 0.150808 & 5.0 \\
HD 216143 & 5 & 742 & -115.99 & 0.23 & 0.51 & 0.43 & 1.18 & 5.72 & 0.221185 & 5.5 \\
HD 218620 & 5 & 749 & -52.34 & 0.33 & 0.73 & 0.38 & 1.91 & 16.62 & 0.002294 & 5.2 \\
HD 218857 & 9 & 7648 & -170.39 & 0.27 & 0.82 & 0.54 & 1.54 & 18.91 & 0.015356 & 2.1 \\
& & & & & & & & & & \\
\hline
\end{tabular}




\section{REFERENCES}

Alonso, A., Arribas, S., \& Martinez Roger, C. 1994, A\&AS, 107, 365

Alonso, A., Arribas, S., \& Martinez Roger, C. 1998, A\&AS, 131, 209

Alonso, A., Arribas, S., \& Martinez Roger, C. 1999, A\&AS, 140, 261

Alonso, A., Arribas, S., \& Martinez Roger, C. 2001, A\&A, 376, 1039

Anthony-Twarog, B. J., \& Twarog, B. A. 1994, AJ, 107, 1577 (ATT)

Arribas, S., \& Martinez Roger, C. 1987, A\&AS, 70, 303

Behr, B. 2003, ApJS, 149, 101

Behr, B. B., Cohen, J. G., \& McCarthy, J. K. 2000, ApJ, 531, L37

Behr, B. B., Djorgovski, S. G., Cohen, J. G., McCarthy, J. K., Côté, P., Piotto, G., \& Zoccalo, M. 2000, ApJ, 528, 849

Bond, H. E. 1980, ApJS, 44, 517

Bopp, B. W., \& Stencel, R. E. 1981, ApJ, 247, L31

Brown, J. A., \& Wallerstein, G. 1992, AJ, 104, 1818

Burris, D. L., Pilachowski, C. A., Armandroff, T. E., Sneden, C., Cowan J. J., \& Roe, H. 2000, ApJ, 544, 302

Carney, B. W. 1980, AJ, 85, 38

Carney, B. W. 1983, AJ, 88, 623

Carney, B. W., Gray, D. F., Yong, D., Latham, D. W., Manset, N., Zelman, R., \& Laird, J. B. 2007 , in preparation

Carney, B. W., \& Latham, D. W. 1986, AJ, 92, 60 (C\&L)

Carney, B. W., Latham, D. W., Stefanik, R. P., Laird, J. B., \& Morse, J. A. 2003, AJ, 125, 293 (C2003)

Cohen, J. G., \& McCarthy, J. K. 1997, AJ, 113, 1353

Côté, Djorgovski, S. G., Meylan, G., Castro, S., \& McCarthy, J. K. 2002, ApJ, 574, 782

Côté, P., Pryor, C., McClure, R. D., Fletcher, J. M., \& Hesser, J. E. 1996, AJ, 112,574

de Medeiros, J. R., do Nascimento, Jr., J. D., Sankarankutty, S., Costa, J. M., \& Maia, M. R. G. 2000, A\&A, 363, 239

de Medeiros, J. R., Silva, J. R. P., Do Nascimento, J. D., Jr., Canto Martins, B. L., da Silva, L., Melo, C., \& Burnet, M. 2006, A\&A, 458, 895 
Fernie, J. D. 1983, PASP, 95, 782

Fulbright, J. A. 2000, AJ, 120, 1841

Goldberg, D., Mazeh, T., Latham, D. W., Stefanik, R. P., Carney, B. W., \& Laird, J. B. 2002, AJ, 124, 1132

Gratton, R. G., Carretta, E., \& Castelli, F. 1996, A\&A, 314, 191

Gray, D. F. 1982, ApJ, 262, 282

Gray, D. F. 1984, ApJ, 281, 719

Gray, D. F., \& Pallavicini, R. 1989, PASP, 101, 685

Gray, D. F., \& Toner, C. G. 1986, ApJ, 310, 277

Gray, D. F., \& Toner, C. G. 1987, ApJ, 322, 360

Gunn, J. E., \& Griffin, R. F. 1979, AJ, 84, 752

Kinman, T., Castelli, F., Cacciari, C., Bragaglia, A., Harmer, D., \& Valdes, F. 2000, A\&A, 364,102

Kraft, R. P., \& Ivans, I. I. 2003, PASP, 115, 143

Kraft, R. P., Sneden, C., Langer, G. E., \& Prosser, C. F. 1992, AJ, 104, 645

Kraft, R. P., Sneden, C., Smith, G. H., Shetrone, M. D., Langer, G. E., \& Pilachowski, C. A. 1997, AJ, 113, 279

Kurtz, M. J., \& Mink, D. J. 1998, PASP, 110, 934

Kurucz, R. L., Furenlid, I. Brault, J., \& Testerman, L. 1984. Solar Flux Atlas from 296 to 1300 nm, (National Solar Observatory, Sunspot, NM)

Latham, D. W. 1985, in Stellar Radial Velocities, IAU Coll. No. 88, ed. A. G. D. Philip \& D. W. Latham (L. Davis Press, Schenectady), p. 5

Latham, D. W. 1992, in Complementary Approaches to Binary and Multiple Star Research, IAU Coll. No. 135, ed. H. McAlister \& W. Hartkopf (ASP, San Francisco), p. 110

Latham, D. W., Stefanik, R. P., Torres, G., Davis, R. J., Mazeh, T., Carney, B. W., Laird, J. B., \& Morse, J. A. 2002, AJ, 124, 1144

Lupton, R. H., Gunn, J. E., \& Griffin, R. F. 1987, AJ, 93, 114

Massarotti, A., Latham D. W., Stefanik, R. P., Fogel, J., Carney, B. W., \& Laird, J. B. 2007, in preparation

Mayor, M., \& Mermilliod, J.-C. 1984, in Observational Tests of Stellar Evolution Theory, IAU Sym. No. 105, ed. A. Maeder \& A. Renzini (Reidel, Dordrecht), p. 411 
Mayor, M., Meylan, G., Udry, S., Duquennoy, A., Andersen, J., Nordström, B., Imbert, M., Maurice, M., Prévot, L., Ardeberg, A., \& Lindgren, H. 1997, AJ, 114, 1087

Nordström, B., Latham, D. W., Morse, J. A., Milone, A. A. E., Kurucz, R. L., Andersen, J., \& Stefanik, R. P. 1994, A\&A, 287, 338

Norris, J., Bessell, M. S., \& Pickles, A. J. 1985, ApJS, 58, 463 (NBP)

Peterson, R. C. 1983, ApJ, 275, 737

Peterson, R. C. 1985, ApJ, 289, 320

Peterson, R. C., Rood, R. T., \& Crocker, D. A. 1995, ApJ, 453, 214

Peterson, R. C., Tarbell, T. D., \& Carney, B. W. 1983, ApJ, 265, 972

Pryor, C., Latham D. W., \& Hazen, M. L. 1988, AJ, 96, 123

Recio-Blanco, A., Piotto, G., Aparicio, A., \& Renzini, A. 2002, astro-ph 0204403

Siess, L., \& Livio, M. 1999, MNRAS, 308, 1133

Skrutskie, M. F. et al. 2006, AJ, 131, 1163

Soker, N. 1998, AJ, 116, 1308

Sozzetti, A., Torres, G., Latham, D. W., Carney, B. W., Stefanik, R. P., Boss, A. P., \& Laird, J. B. 2006, ApJ, 649, 428

Stefanik, R. P., Latham, D. W., \& Torres, G. 1999, in Precise Radial Velocities, ASP Conf. Series No. 185, ed. J. B. Hearnshaw \& C. D. Scarfe (ASP, San Francisco), p. 354

Stone, R. P. S. 1983, PASP, 95, 27

Straniero, O., \& Chieffi, A. 1991, ApJS, 76, 525 


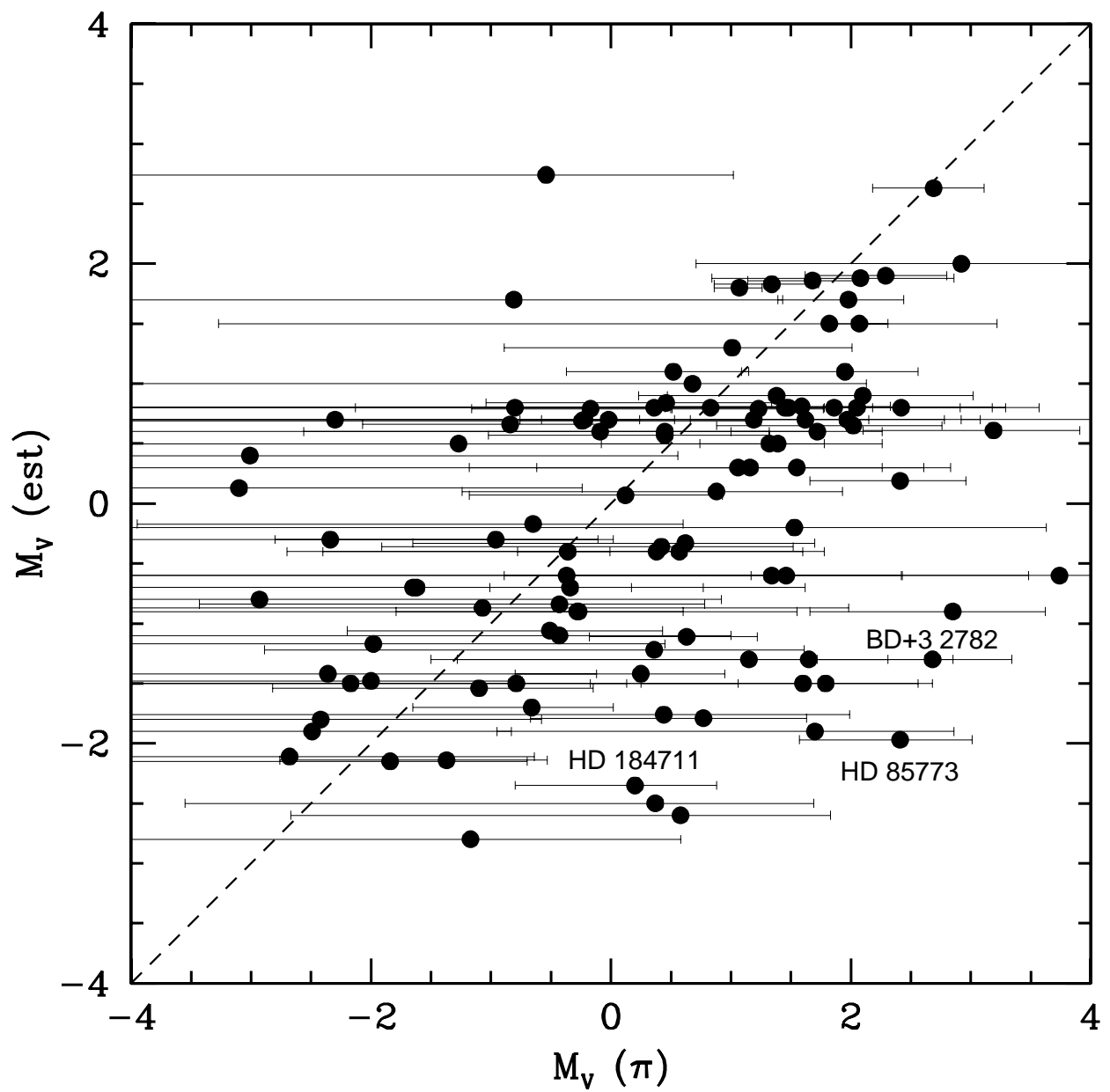

Fig. 1.- A comparison of our estimated $M_{\mathrm{V}}$ values with those derived using HIPPARCOS parallaxes. Stars with negative parallaxes have been omitted, and were stars with $M_{\mathrm{V}} \leq$ -4.0. Three stars are flagged and discussed in the text. 


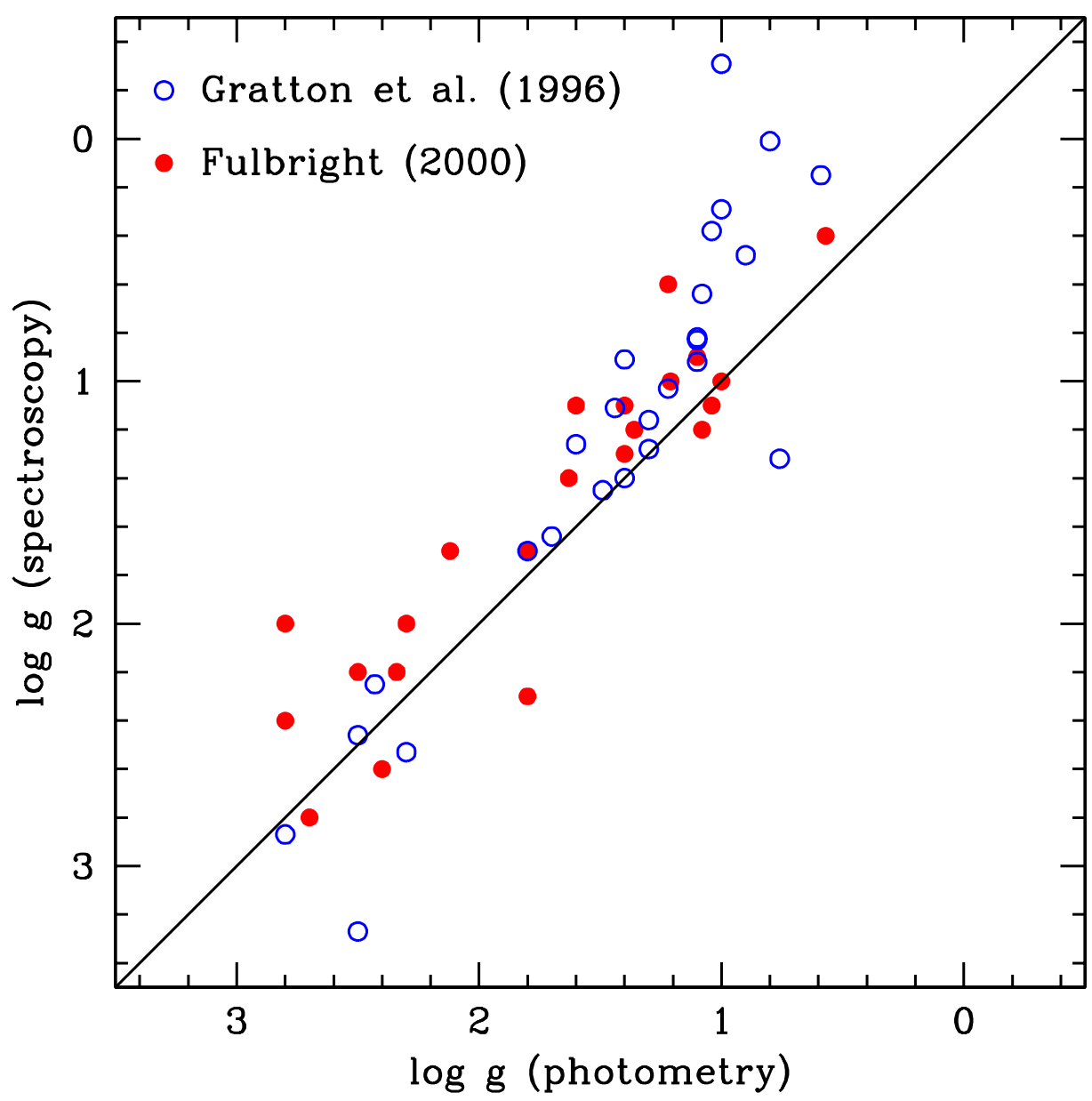

Fig. 2.- A comparison of the gravities derived from photometry with those obtained spectroscopically by Gratton et al. (1996) and Fulbright (2000). 


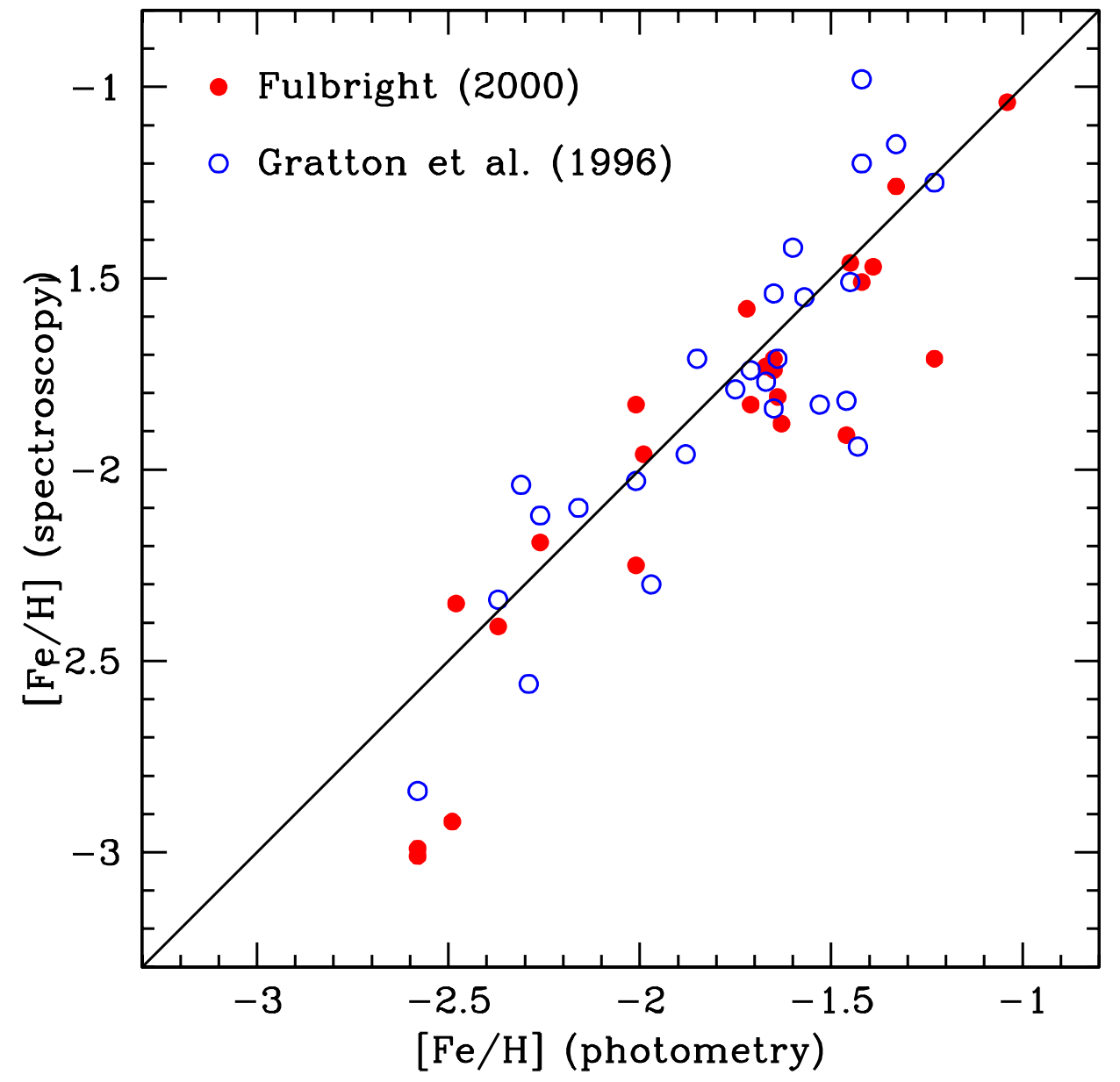

Fig. 3.- A comparsison of our adopted photometric metallicities with those obtained spectroscopically by Gratton et al. (1996) and Fulbright (2000). 


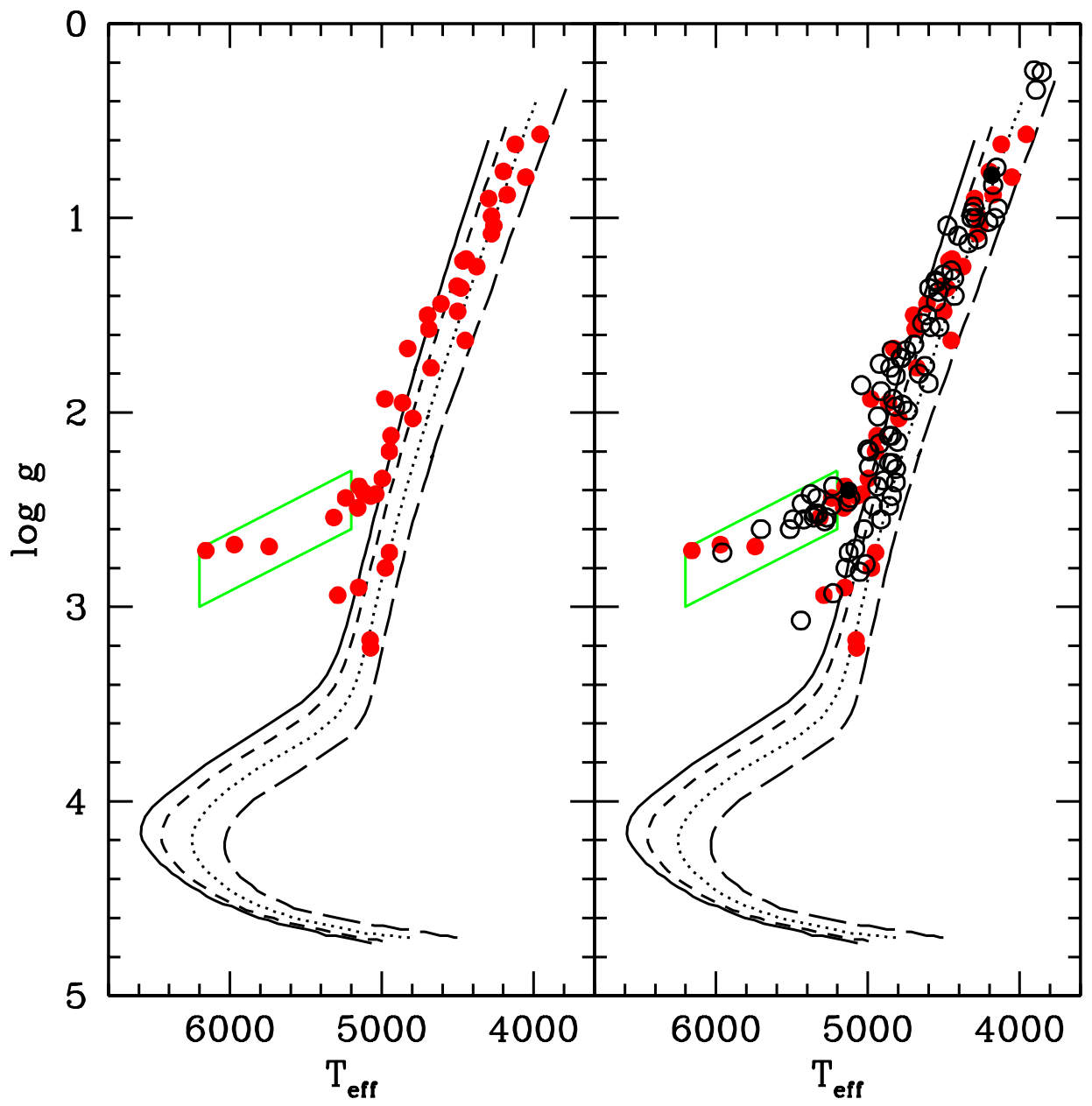

Fig. 4.- A comparison between the gravities and temperatures estimated for our new program stars (left panel) and the combined samples from this paper and C2003 (right panel). The model isochrones are taken from Straniero \& Chieffi (1991), with Z $=0.0001$, 0.0003, 0.001, and 0.003. The more metal-poor isochrones lie at higher temperatures. The adopted age in all cases is 14 Gyrs. Open circles denote the C2003 program stars. The parallelograms enclose the red horizontal branch stars. 

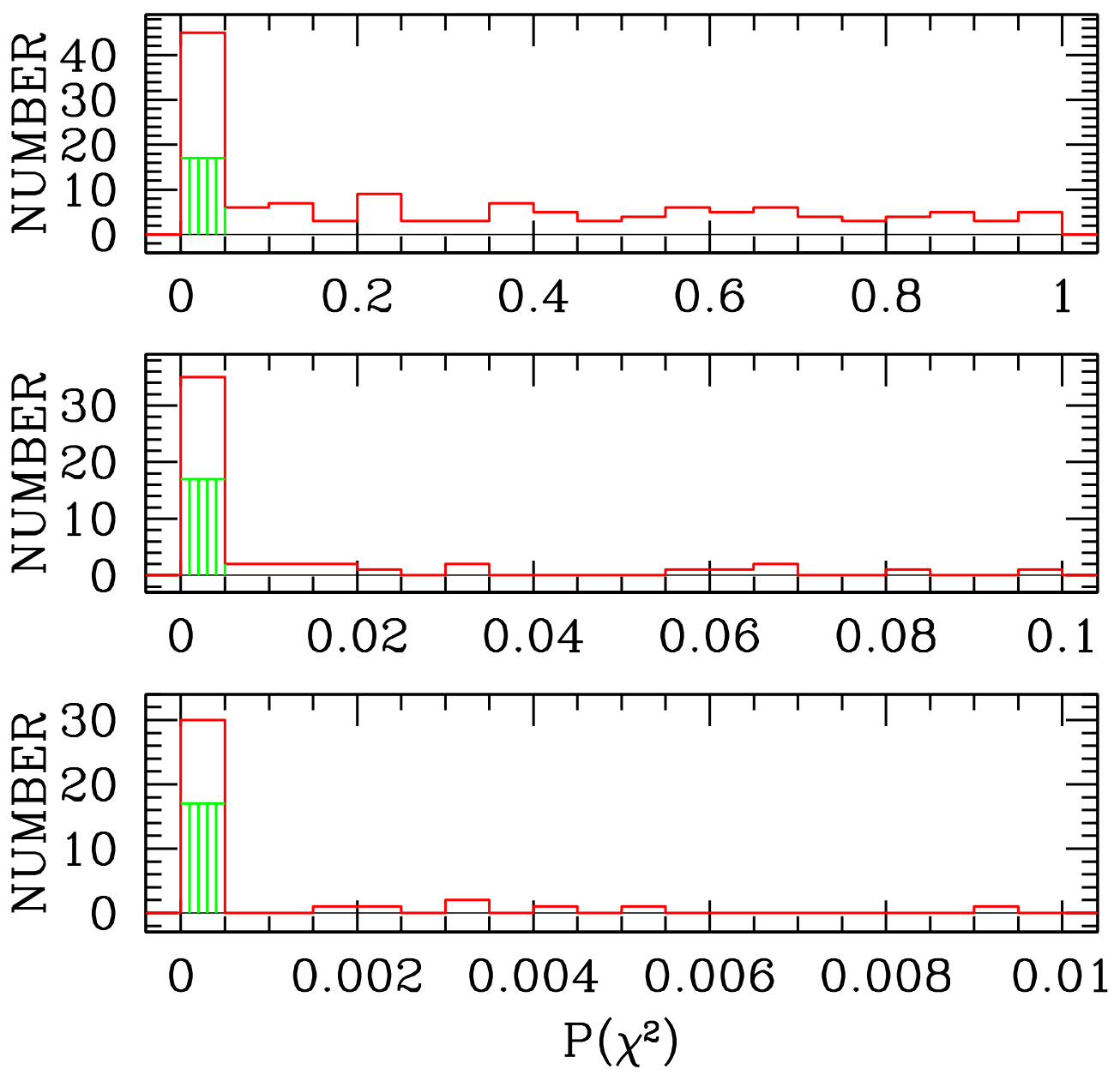

Fig. 5.- The distribution of the probabilities of $\chi^{2}$ for our program stars, excluding the three spectroscopic binaries for which orbital solutions are not yet available (HD 4306, BD-1 2582, and HD 108317). There is an excess of small values, indicating a substantial number of stars with variable velocities. Vertical shading indicates stars found to be spectroscopic binaries. 

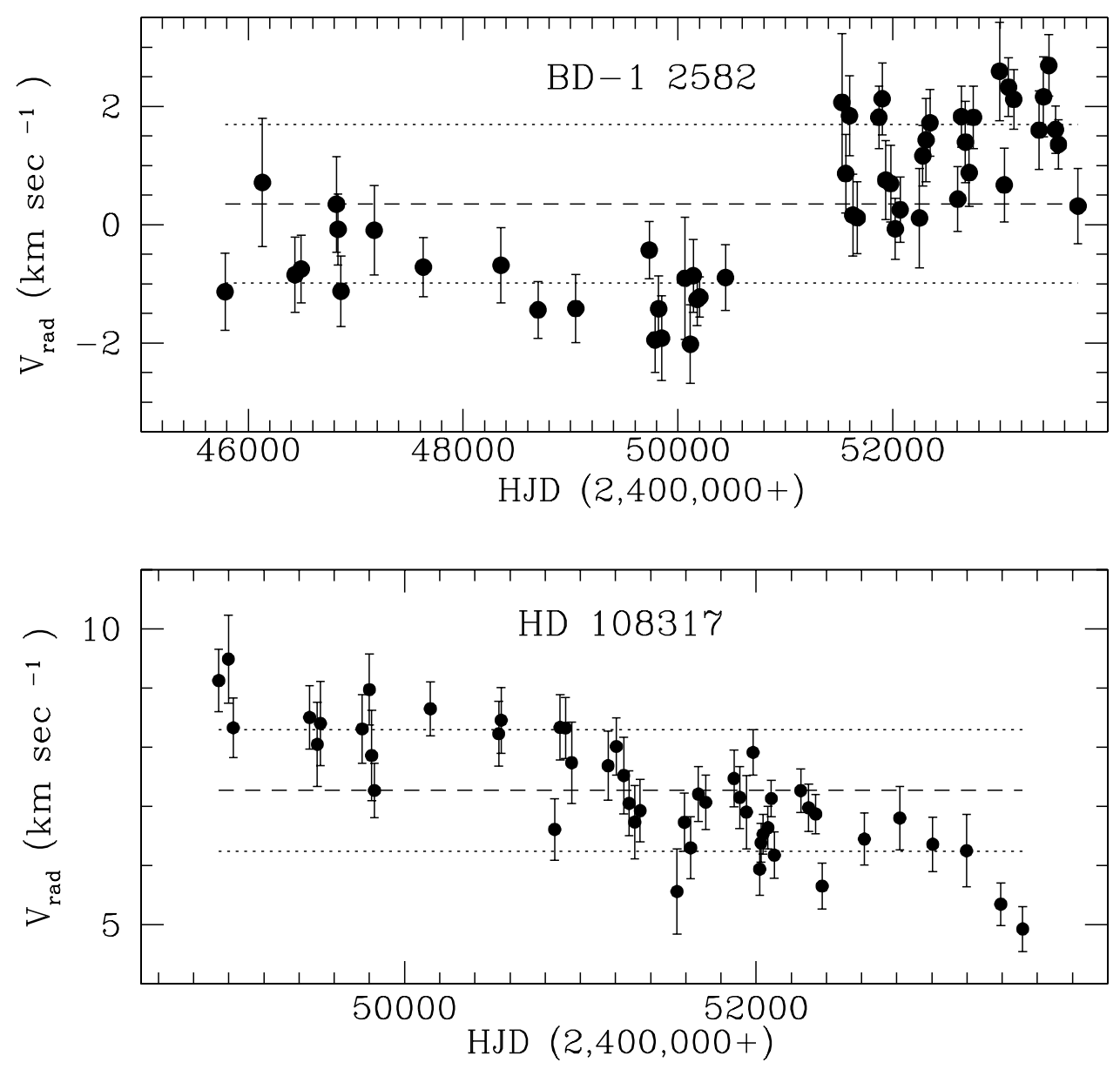

Fig. 6. - The radial velocity histories of two binary stars from C2003, for which we have obtained additional observations, but whose orbital solutions are as yet incomplete: (a) BD-1 2582, and (b) HD 108317. The mean velocities as well as $\pm 1 \sigma_{\text {int }}$ are also shown as horizontal lines. 

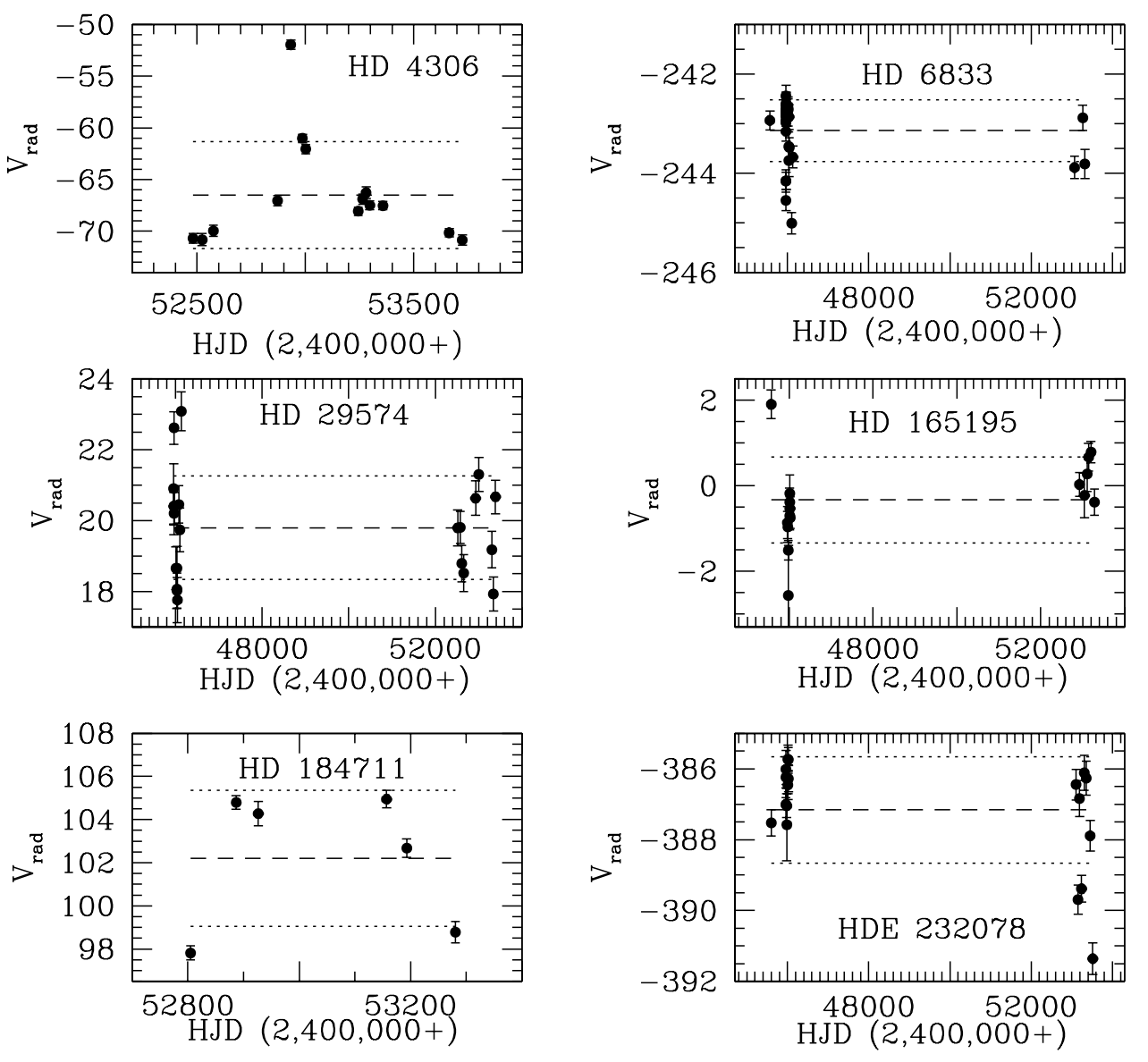

Fig. 7.- The velocity histories, in $\mathrm{km} \mathrm{s}^{-1}$, of the six stars in our new study with $\mathrm{P}\left(\chi^{2}\right)$ $\leq$ 0.001. (a) HD 4306; (b) HD 6833; (c) HD 27295; (d) HD 165195; (e) HD 184711; and (f) HDE 232078. The mean velocities as well as $\pm 1 \sigma_{\text {int }}$ are also shown as horizontal lines. 


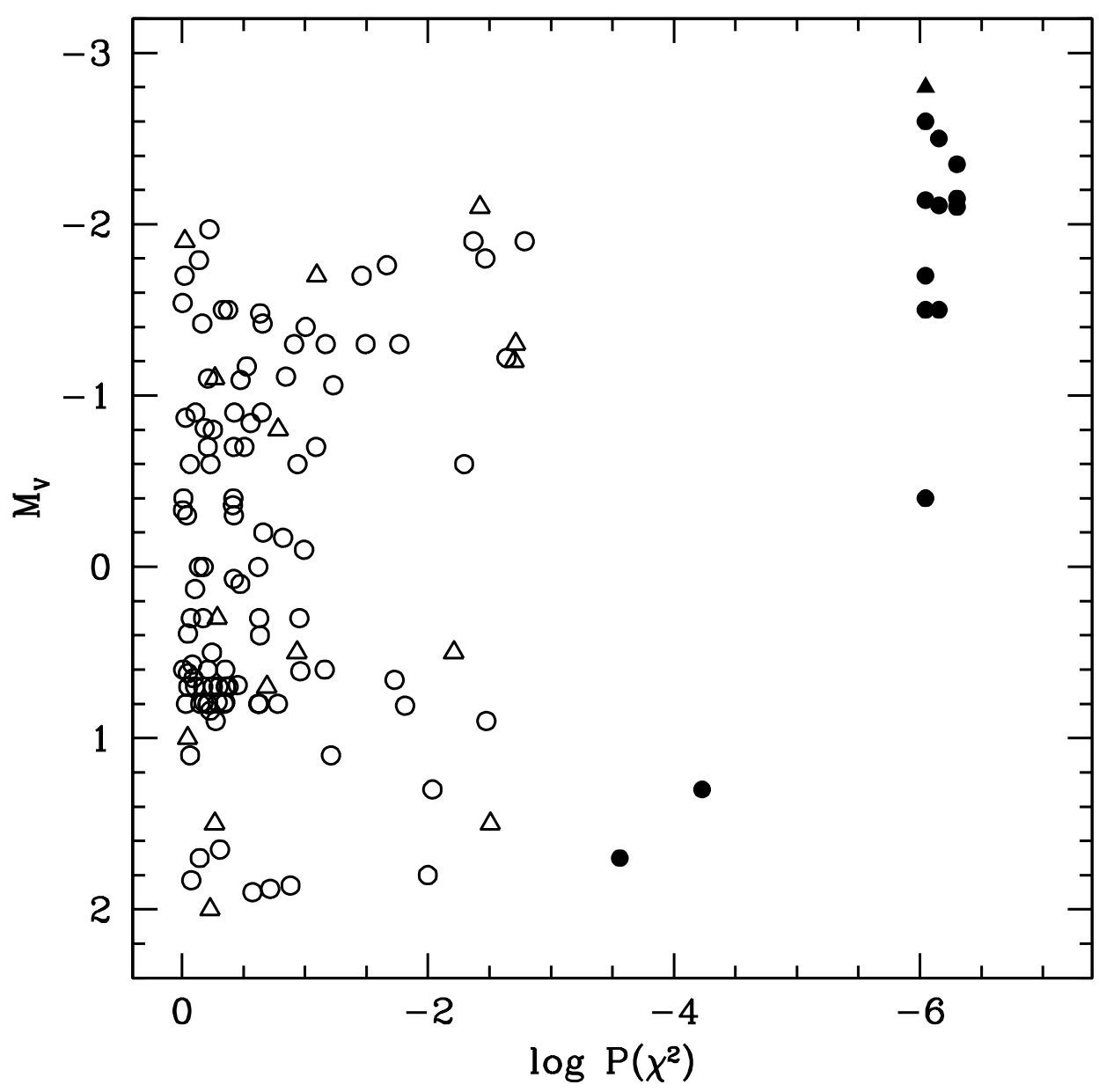

Fig. 8.- The logarithms of the $\mathrm{P}\left(\chi^{2}\right)$ values are plotted against the estimated absolute magnitudes. Non-binary stars with $\mathrm{P}\left(\chi^{2}\right) \leq 0.001$ are plotted as filled circles and are assumed to be displaying velocity jitter. All other non-binary stars are plotted as open circles. The spectroscopic binary stars' $\mathrm{P}\left(\chi^{2}\right)$ values have been computed using the residuals from the orbital solutions. The binary stars are represented by triangles. 


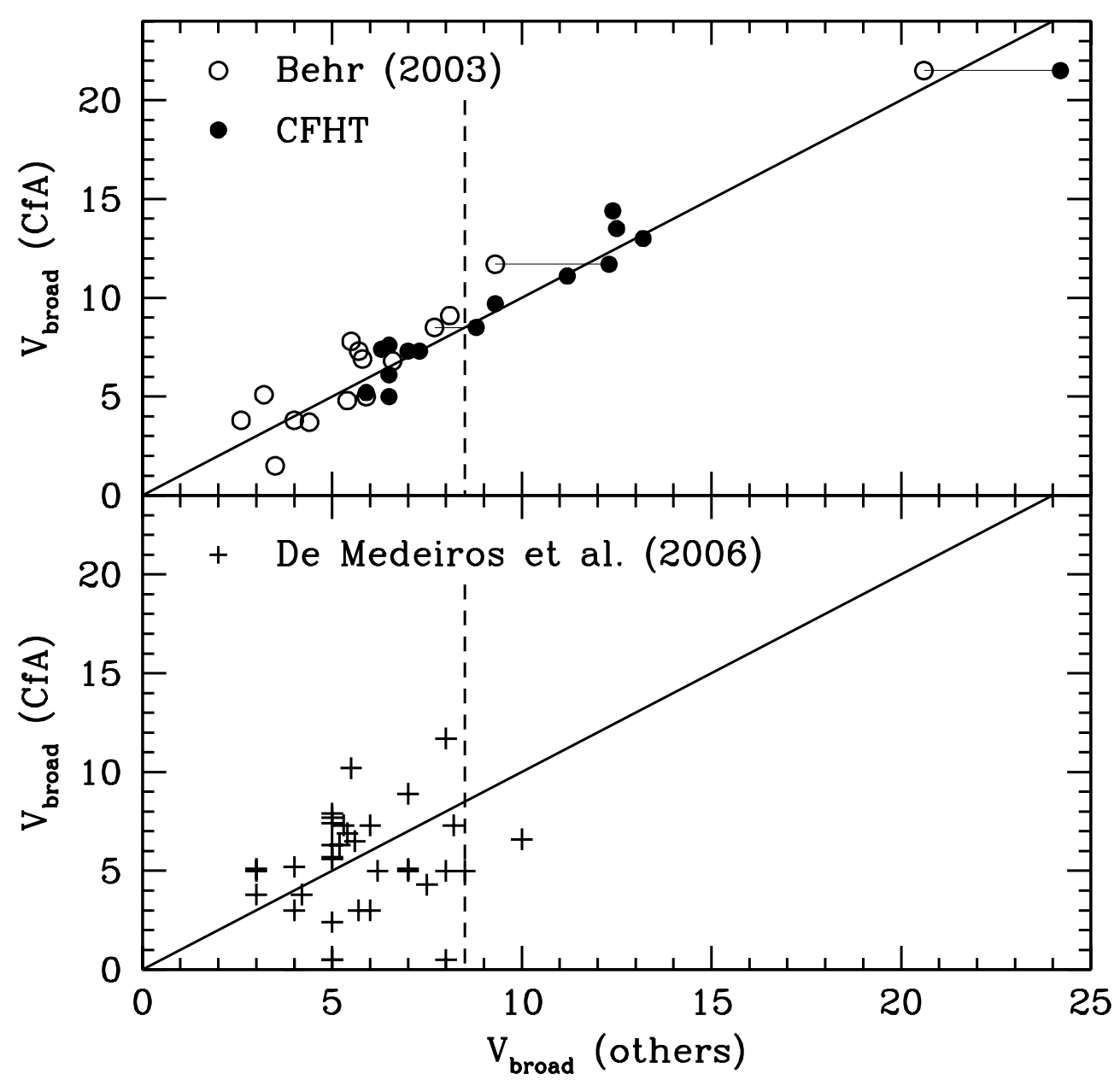

Fig. 9.- A comparison of our derived line broadening measures, in $\mathrm{km} \mathrm{s}^{-1}$, with those of Behr (2003), de Medeiros et al. (2006), and our own very high-resolution, high-S/N spectra obtained using the Gecko spectrograph at CFHT. The diagonal line is not a fit, but a unit slope. The vertical dashed line represents the instrumental resolution of the CfA equipment, about $8.5 \mathrm{~km} \mathrm{~s}^{-1}$. Thin solid lines connect the two measures obtained for three stars from Behr (2003) and our CFHT spectra. 


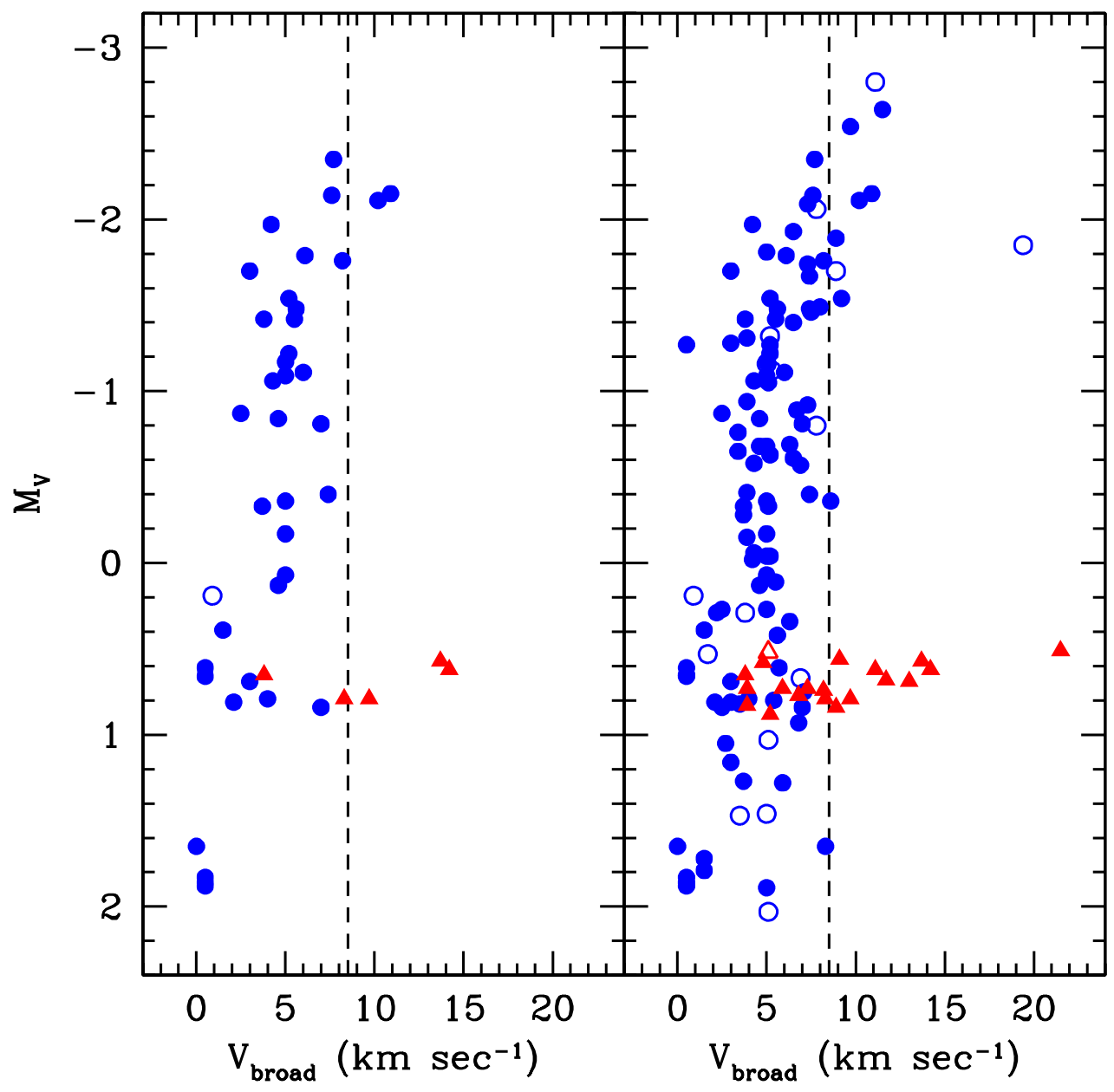

Fig. 10. - The estimated line broadening in the spectra of the new program stars (left), and the combined results from this program and C2003 (right). Unlike the similar figure in C2003, we have plotted HD 3008 and BD+22 2411 with their derived $V_{\text {broad values. Open }}$ blue circles identify the red giant binaries in the sample. Filled red triangles are used to show the red horizontal branch stars enclosed within the parallelogram of Figure 3. (The open red triangle is the red horizontal branch binary star, HD 108317.) The dashed line indicates our instrumental resolution of $8.5 \mathrm{~km} \mathrm{~s}^{-1}$. 


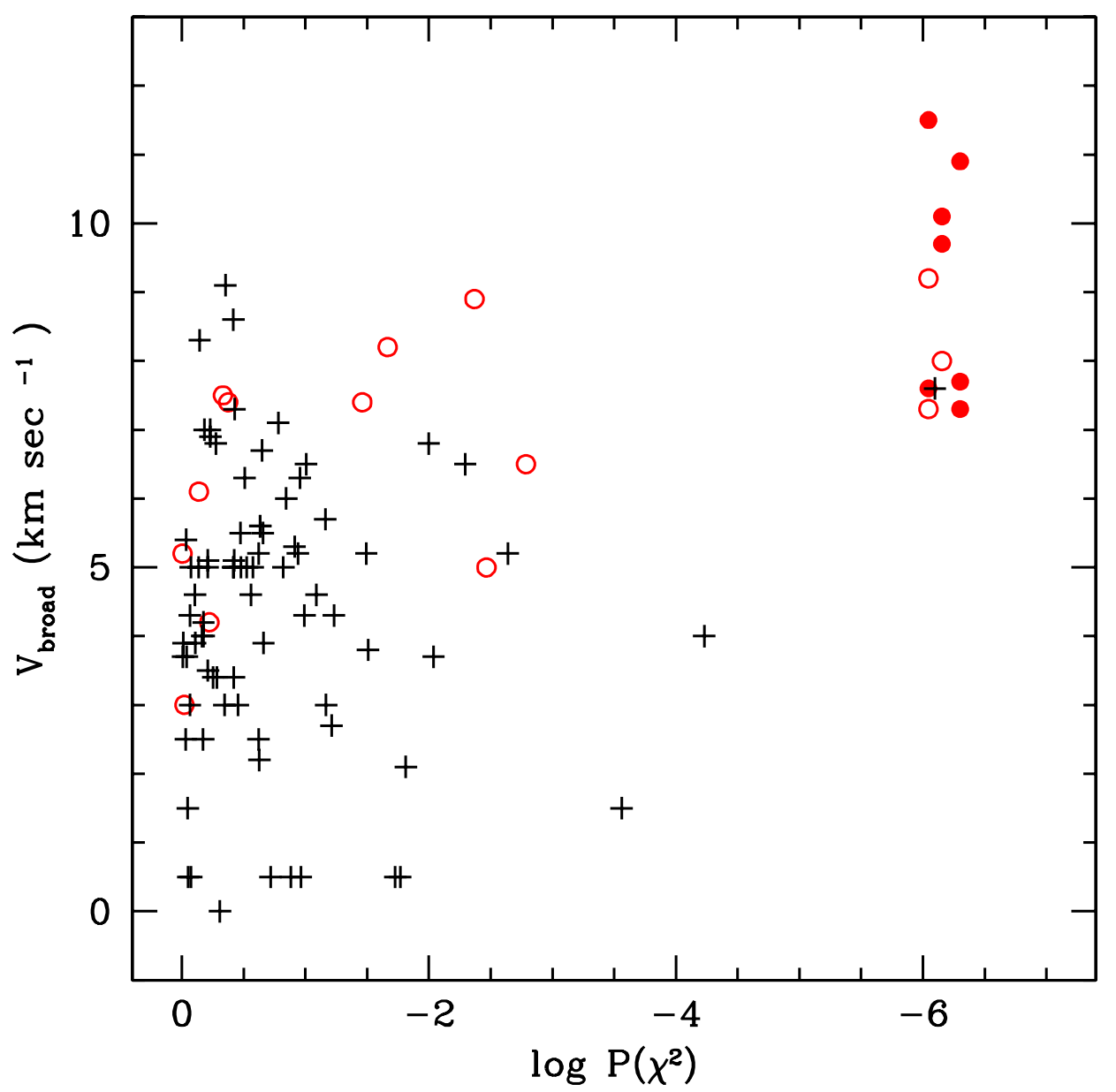

Fig. 11.- The logarithm of the probability of $\chi^{2}$ is compared with the measured line broadening, $V_{\text {broad. }}$ Stars with $M_{\mathrm{V}} \leq-2.0$ are shown as filled red circles, and those with $-2.0<M_{\mathrm{V}} \leq-1.5$ are shown as open red circles. 


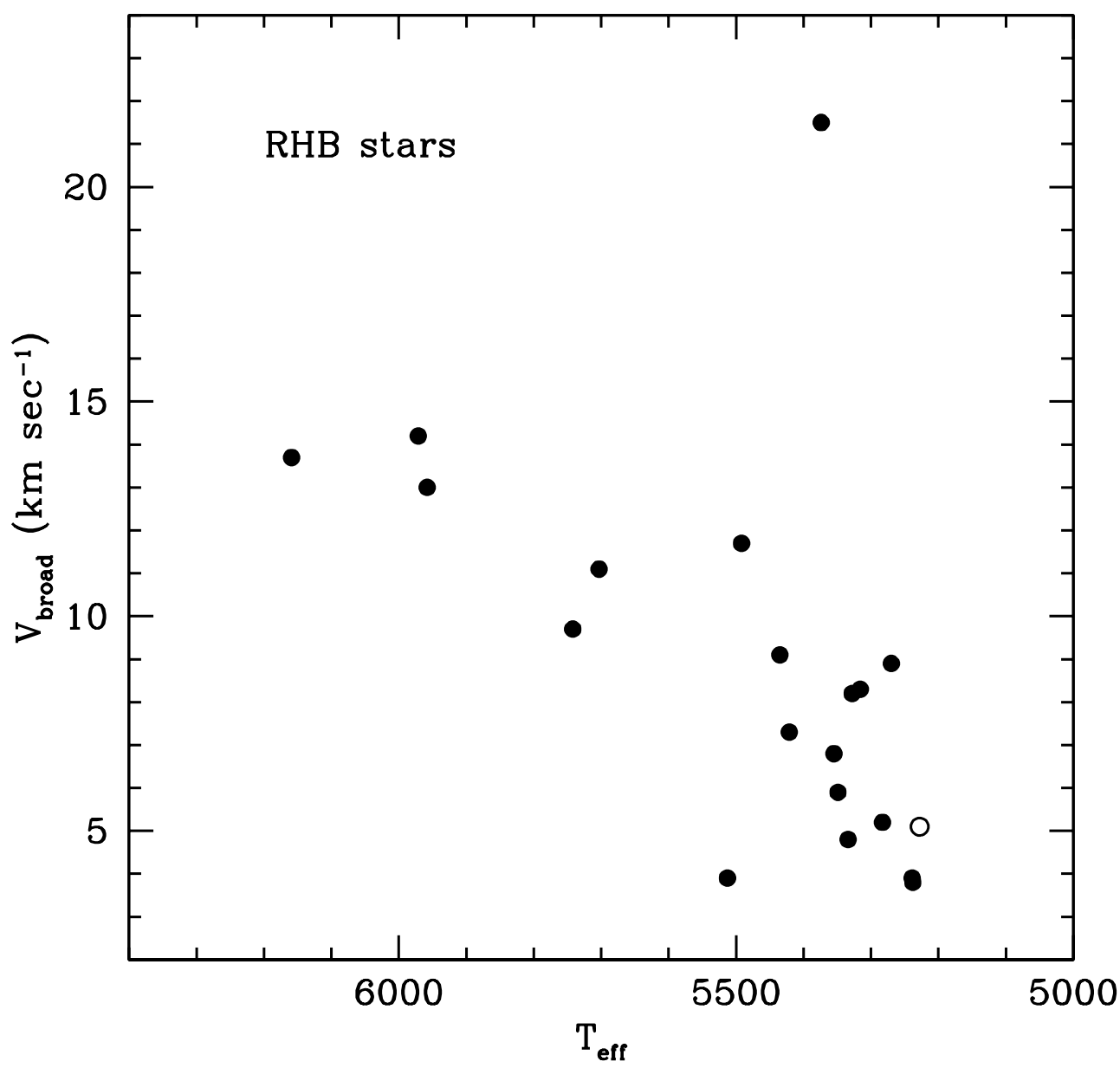

Fig. 12.- The measured line broadening is compared to the estimated effective temperature of the red horizontal branch stars in this study and that of C2003. Macroturbulence is expected to increase with temperature at constant luminosity. The open circle is the longperiod spectroscopic binary HD 108317. 